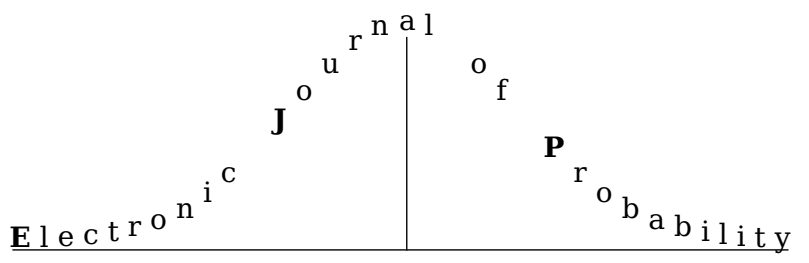

Electron. J. Probab. 24 (2019), no. 109, 1-25.

ISSN: 1083-6489 https://doi.org/10.1214/19-EJP367

\title{
Decompositions of infinitely divisible nonnegative processes
}

\author{
Nathalie Eisenbaum*
}

\begin{abstract}
We establish decomposition formulas for nonnegative infinitely divisible processes. They allow to give an explicit expression of their Lévy measure. In the special case of infinitely divisible permanental processes, one of these decompositions represents a new isomorphism theorem involving the local time process of a transient Markov process. We obtain in this case the expression of the Lévy measure of the total local time process which is in itself a new result on the local time process. Finally, we identify a determining property of the local times for their connection with permanental processes.
\end{abstract}

Keywords: infinitely divisible process; Lévy measure; permanental process; local time; Markov process; Gaussian process.

AMS MSC 2010: 60E07; 60G15; 69G17; 60G51; 60J25; 60J55.

Submitted to EJP on October 25, 2018, final version accepted on September 18, 2019.

\section{Introduction and main results}

A real valued process $\psi=\left(\psi_{x}, x \in E\right)$ indexed by a general set $E$, is infinitely divisible if all its finite dimensional marginals are infinitely divisible. According to the Lévy-Khintchine formula, for every $n$, and every $x_{1}, . ., x_{n}$ in $E$, the $n$-dimensional marginal $\left(\psi_{x_{1}}, \psi_{x_{2}}, \ldots, \psi_{x_{n}}\right)$ admits a decomposition into three independent vectors: one deterministic vector, one centered Gaussian vector and one vector whose law is characterized by a Lévy measure $\nu_{\left(x_{1}, \ldots, x_{n}\right)}$ on $\mathbb{R}^{n}$.

We assume that $\psi$ is nonnegative, hence the Gaussian component is always reduced to 0 . We also assume that $\psi$ has no drift which implies that the deterministic component is also always nul. Moreover the Lévy measure $\nu_{\left(x_{1}, \ldots, x_{n}\right)}$ must be on $\mathbb{R}_{+}^{n}$.

What is known about $\nu_{\left(x_{1}, . ., x_{n}\right)}$ ? According to its definition, it must satisfy for every $\alpha_{1}, . ., \alpha_{n}$ in $\mathbb{R}_{+}^{n}$

$$
\mathbb{E}\left[\exp \left\{-\sum_{i=1}^{n} \alpha_{i} \psi_{x_{i}}\right\}\right]=\exp \left\{-\int_{\mathbb{R}_{+}^{n}}\left(1-e^{-\sum_{i=1}^{n} \alpha_{i} t_{i}}\right) \nu_{\left(x_{1}, \ldots, x_{n}\right)}(d t)\right\},
$$

\footnotetext{
${ }^{*}$ CNRS and Université Paris Descartes, MAP5, France. E-mail: nathalie. eisenbaum@parisdescartes.fr
} 
with $\nu_{\left(x_{1}, . ., x_{n}\right)}\left(0_{\mathbb{R}^{n}}\right)=0$, and $\int_{\mathbb{R}_{+}^{n}} 1 \wedge|t| \nu_{\left(x_{1}, . ., x_{n}\right)}(d t)<\infty$, where $|t|=\sup _{1 \leq i \leq n}\left|t_{i}\right|$.

But in general, nothing else is known about $\nu_{\left(x_{1}, \ldots, x_{n}\right)}$. Given an infinitely divisible process $\psi$, the first natural problem is to determine $\left\{\nu_{\left(x_{1}, . ., x_{n}\right)},\left(x_{1}, . ., x_{n}\right) \in E^{n}, n>0\right\}$. One can formulate the problem in a more concise way by using the existence of a unique global Lévy measure $\nu$ on $\mathbb{R}_{+}^{E}$, the space of all functions from $E$ into $\mathbb{R}_{+}$, (Theorem 2.8 in [16]) such that for every $n>0$ and every $x_{1}, . ., x_{n}$ in $E$ :

$$
\mathbb{E}\left[\exp \left\{-\sum_{i=1}^{n} \alpha_{i} \psi_{x_{i}}\right\}\right]=\exp \left\{-\int_{\mathbb{R}_{+}^{E}}\left(1-e^{-\sum_{i=1}^{n} \alpha_{i} y\left(x_{i}\right)}\right) \nu(d y)\right\},
$$

where for $y$ element of $\mathbb{R}_{+}^{E}$ and $x$ in $E, y(x)$ denotes the image of $x$ by the function $y$.

The measure $\nu$ is called the Lévy measure of the process $\psi$ (in section 2, we detail this result due to Rosinski [16]). The problem becomes to know the Lévy measure $\nu$.

Under an assumption of stochastic continuity, we give in Theorem 1.2 below, the general expression of the Lévy measure of nonnegative infinitely divisible processes without drift. To obtain it, we will first establish decomposition formulas of the nonnegative infinitely divisible processes (Theorems 1.1 and 1.3) and use a previously established general isomorphism theorem [5].

In the particular case of infinitely divisible permanental processes, the expression of the Lévy measure was already known. But surprisingly, as it will be highlighted in Remark 1.7, this example reflects the precise form of the Lévy measure in the general case.

Theorem 1.1. Let $\left(\psi_{x}, x \in E\right)$ be a nonnegative infinitely divisible process with no drift part and finite first moment. Then for every $a$ in $E$ such that $\mathbb{E}[\psi(a)]>0$, the process $\left(\psi_{x}, x \in E \mid \psi_{a}=0\right)$ is infinitely divisible and there exists a nonnegative infinitely divisible process $\left(\mathcal{L}_{x}^{(a)}, x \in E\right)$, independent of $\left(\psi_{x}, x \in E \mid \psi_{a}=0\right)$ such that:

$$
\psi^{(\text {law })}=\left(\psi \mid \psi_{a}=0\right)+\mathcal{L}^{(a)} .
$$

The three processes involved in Theorem 1.1 are infinitely divisible. Hence Theorem 1.1 has a counterpart in terms of Lévy measures. To formulate it we use a family of nonnegative processes associated to $\psi$ (see [5]) in the following way:

For every a such that $\mathbb{E}[\psi(a)]>0$, there exists a nonnegative process $\left(r^{(a)}(x), x \in E\right)$ independent of $\psi$ such that

$$
\psi+r^{(a)} \text { has the law of } \psi \text { under } \mathbb{E}\left[\frac{\psi(a)}{\mathbb{E}[\psi(a)]}, .\right]
$$

Actually the existence of $\left(r^{(a)}, a \in E\right)$ characterizes the infinite divisiblity of $\psi$. This characterization has been established in [5] (see also [16] for a more general framework).

When $E$ is assumed to be a separable metric space w.r.t. some metric $d$, a real valued process $(Y(x), x \in E)$ is stochastically continuous if for every $\varepsilon>0$ and every $a$ in $E$

$$
\lim _{x \rightarrow a} \mathbb{P}[|Y(x)-Y(a)|>\varepsilon]=0
$$

where the convergence to $a$ is with respect to the metric $d$.

Theorem 1.2. Let $\left(\psi_{x}, x \in E\right)$ be a nonnegative infinitely divisible process with Lévy mesure $\mu$ and no drift. Denote by $\mu_{a}$ and $\tilde{\mu}_{a}$ the respective Lévy measures of $\left(\psi \mid \psi_{a}=0\right)$ and $\mathcal{L}^{(a)}$. Then we have:

$$
\mu=\mu_{a}+\tilde{\mu}_{a}
$$

where

$$
\mu_{a}(d y)=1_{\{y(a)=0\}} \mu(d y), \quad \tilde{\mu}_{a}(d y)=1_{\{y(a)>0\}} \mu(d y),
$$


and for any measurable functional $F$ on $\mathbb{R}_{+}^{E}$

$$
\tilde{\mu}_{a}(F)=\mathbb{E}\left[\frac{\mathbb{E}[\psi(a)]}{r^{(a)}(a)} F\left(r^{(a)}\right)\right] .
$$

If one assumes moreover that $E$ is a separable metric space and that $\psi$ is stochastically continuous then for any $\sigma$-finite measure $m$ with support equal to $E$ such that $\int_{E} \mathbb{E}[\psi(x)] m(d x)<\infty$ :

$$
\mu(F)=\int_{E} \mathbb{E}\left[\frac{F\left(r^{(a)}\right)}{\int_{E} r^{(a)}(x) m(d x)}\right] \mathbb{E}[\psi(a)] m(d a) .
$$

At first sight, the fact that the expression (1.2) of the Lévy measure of $\psi$ is independent of the choice of the measure $m$, is remarkable. As we will show in Remark 2.2, this fact can be justified by the basic properties of the family $\left(r^{(a)}, a \in E\right)$.

To exploit (1.2), one needs to know the law of $r^{(a)}$ for every $a$ such that $\mathbb{E}[\psi(a)]>0$. In section 4 we show how to proceed in the case of squared Bessel processes by making use of the Markov property.

The following theorem can be viewed as an extension of Theorems 1.1 and 1.2 which correspond to the case of the Dirac measure at point $a$. Instead of a Dirac measure we consider a $\sigma$-additive measure $m$ on $E$.

Theorem 1.3. Let $\left(\psi_{x}, x \in E\right)$ be a nonnegative infinitely divisible process with no drift. Let $m$ be a $\sigma$-additive measure on $E$ and $V$ be an open subset of the support of $m$ such that $\int_{V} \mathbb{E}[\psi(x)] m(d x)<\infty$. Denote by $\phi$ the process $\psi$ conditioned on $\int_{V} \psi(x) m(d x)=0$.

Then $\phi$ is infinitely divisible and there exists an infinitely divisible nonnegative process $\left(\mathcal{L}_{x}^{V}, x \in E\right)$ independent of $\phi$ such that

$$
\psi \stackrel{(\text { law })}{=} \phi+\mathcal{L}^{V} .
$$

Denote by $\mu, \mu_{V}$ and $\tilde{\mu}_{V}$ the respective Lévy measure of $\left(\psi, \int_{V} \psi(x) m(d x)\right)$, $\left(\phi, \int_{V} \phi(x) m(d x)\right)$ and $\left(\mathcal{L}^{V}, \int_{V} \mathcal{L}_{x}^{V} m(d x)\right)$, then we have:

$$
\mu=\mu_{V}+\tilde{\mu}_{V}
$$

with

$$
\mu_{V}(d y d t)=\mu(d y \times\{0\}), \tilde{\mu}_{V}(d y d t)=1_{t>0} \mu(d y d t),
$$

and for any measurable functional $F$ on $\mathbb{R}_{+}^{E} \times \mathbb{R}_{+}$

$$
\tilde{\mu}_{V}(F)=\int_{V} \mathbb{E}\left[\frac{F\left(r^{(a)}, \int_{V} r_{x}^{(a)} m(d x)\right)}{\int_{V} r_{x}^{(a)} m(d x)}\right] \mathbb{E}[\psi(a)] m(d a) .
$$

If moreover, $E$ is a separable metric space and $\psi$ is stochastically continuous then we have:

$$
\psi_{\left.\right|_{V}} \stackrel{(\text { law) }}{=} \mathcal{L}_{\left.\right|_{V}}^{V} .
$$

In the case when the infinitely divisible process is a permanental process, the above decompositions can be more explicit. To present them, we first recall that a permanental process $(\phi(x), x \in E)$ with index $\beta>0$ and a kernel $k=(k(x, y),(x, y) \in E \times E)$ is characterized by its finite dimensional Laplace transforms:

$$
\mathbb{E}\left[\exp \left\{-\frac{1}{2} \sum_{i=1}^{n} \alpha_{i} \phi\left(x_{i}\right)\right\}\right]=\operatorname{det}(I+\alpha K)^{-1 / \beta}
$$


where $\alpha$ is the diagonal matrix with diagonal entries $\left(\alpha_{i}\right)_{1 \leq i \leq n}, I$ is the $n \times n$-identity matrix and $K$ is the matrix $\left(k\left(x_{i}, x_{j}\right)\right)_{1 \leq i, j \leq n}$.

Note that in case $\beta=2$ and $k$ is symmetric positive definite, $\phi$ equals in law $\left(\eta_{x}^{2}, x \in E\right)$ where $\left(\eta_{x}, x \in E\right)$ is a centered Gaussian process with covariance $k$.

To select infinitely divisible permanental processes, one has to choose an appropriate kernel. To do so, we consider a transient Markov process $X$ with state space $E$, admitting 0 -potential densities $(g(x, y),(x, y) \in E \times E)$ w.r.t. a $\sigma$-finite reference measure $m$ and a local time process $\left(L_{t}^{x}, x \in E, t \geq 0\right)$. More precisely $X$ is a transient Borel right process $\left(\Omega, \mathcal{F},\left(\mathcal{F}_{t}\right),\left(X_{t}\right)_{t \geq 0},\left(\theta_{t}\right), \mathbb{P}_{x}, x \in E\right)$ (where $\mathcal{F}_{t}$ is $\sigma\left\{X_{s}: s \leq t\right\}$ completed and right continuous as usual). To obtain the existence of local times, every point $x$ in the state space $E$ is assumed to be regular for itself that is: $\mathbb{P}_{x}\left(T_{x}=0\right)=1$ where $T_{x}=\inf \left\{t>0: X_{t}=x\right\}$.

We have shown in [7] that there exists an infinitely divisible permanental process with kernel $g$. We have also shown (see [7] and [9]) that a permanental process is infinitely divisible iff it admits for kernel the 0-potential densities of a transient Markov process.

Theorem 1.4. Let $\Psi$ be a permanental process with kernel $g$ and index 1 . For any a in $E$ such that $g(a, a)>0$, denote by $g_{T_{a}}$ the 0-potential densities of $X$ killed at its first hitting time of $a$. Then we have:

$$
\frac{1}{2} \Psi \stackrel{\text { law })}{=} \frac{1}{2} \Psi_{g_{T_{a}}}+L_{\infty}^{(a)},
$$

where $L_{\infty}^{(a)}=\left(L_{\infty}^{(a)}(x), x \in E\right)$ is the total accumulated local times process of $X$ conditioned to start at $a$ and killed at its last visit to $a$, and $\Psi_{g_{T_{a}}}$ is a permanental process with kernel $g_{T_{a}}$ and index 1 , independent of $X$.

In view of Dynkin's isomorphism [2] and its variants and extensions (see [7], [8], [4]...) Theorem 1.4 looks familiar. In section 5, we show how Theorem 1.4 easily generates some of these isomorphism theorems, as well as new identities.

The three processes involved in (1.4) are infinitely divisible. Since the already known isomorphism theorems are expressed in terms of permanental processes with index 2 , denote by $\psi$ a permanental process with kernel $g$ and index 2 and by $\mu$ the Lévy measure of $\frac{1}{2} \psi$. Hence $2 \mu$ is the Lévy measure of $\frac{1}{2} \Psi$. Theorem 1.4 has the following counterpart in terms of Lévy measures. We assume that: $\int_{E} g(x, x) m(d x)<\infty$. We use the notation $\tilde{\mathbb{P}}_{a}$ (and $\tilde{\mathbb{E}}_{a}$ for the corresponding expectation) for the probability under which $X$ starts at $a$ and is killed at its last visit to $a$. This probability is obtained as follows:

for every $\mathcal{F}_{t}$-measurable set $B$

$$
\tilde{\mathbb{P}}_{a}[B]=\frac{1}{g(a, a)} \mathbb{P}_{a}\left[B, g\left(X_{t}, x\right)\right]
$$

Note that the local time process $\left(L_{\infty}^{x}, x \in E\right)$ of $X$ under $\tilde{\mathbb{P}}_{a}$ and the process $\left(L_{\infty}^{(a)}(x), x \in\right.$ $E$ ) have the same law.

The life time of $X$ is denoted by $\zeta$.

Theorem 1.5. Let $\Psi$ be a permanental process with kernel $g$ and index 1 . Let $\Psi_{g_{T_{a}}}$ be a permanental process with kernel $g_{T_{a}}$ and index 1 , and $L_{\infty}^{(a)}$ the total accumulated local times process of $X$ conditioned to start at $a$ and killed at its last visit to $a$. Denote by $2 \mu$ the Lévy measure of $\frac{1}{2} \Psi$. For any $a$ in $E$ such that $g(a, a)>0$

$$
\mu=\mu_{a}+\bar{\mu}_{a}
$$

where $2 \mu_{a}$ is the Lévy measure of $\frac{1}{2} \Psi_{g_{T_{a}}}$ and $2 \bar{\mu}_{a}$ is the Lévy measure of $L_{\infty}^{(a)}$.

Moreover, we have

$$
\mu_{a}(d y)=1_{\{y(a)=0\}} \mu(d y), \quad \bar{\mu}_{a}(d y)=1_{\{y(a)>0\}} \mu(d y),
$$


and for any measurable function $F$ on $\mathbb{R}_{+}^{E}$

$$
\begin{gathered}
\bar{\mu}_{a}(F)=\tilde{\mathbb{E}}_{a}\left[\frac{g(a, a)}{2 L_{\infty}^{a}} F\left(\left(L_{\infty}^{x}, x \in E\right)\right)\right] \\
\mu(F)=\int_{E} \tilde{\mathbb{E}}_{a}\left[\frac{F\left(\left(L_{\infty}^{x}, x \in E\right)\right.}{\zeta}\right] \frac{g(a, a)}{2} m(d a) .
\end{gathered}
$$

Remark 1.6. We mention that in [7], we made a confusion between $\mu$ and $\bar{\mu}_{a}$. This changes the content of Corollary 3.3 in [7] and its consequence Theorem 3.4 [7]. Indeed in case $E$ is a locally compact metric space, the continuity of the local time process obviously implies the continuity of the infinitely divisible process with Lévy measure $2 \bar{\mu}_{a}$ (since it is the local time itself), but this does not immediately imply the continuity of $\psi$. In section 3 , we list some of the properties that $\psi$ must satisfy, assuming the continuity of the local time process. In particular, when $X$ is a transient Lévy process, we show with a simple argument that $\psi$ must be continuous. However we still can not prove that the joint continuity of the local time process implies the continuity of $\psi$ in the general case.

Remark 1.7. Note that unlike in Theorem 1.2, we dont need to assume the stochastic continuity of the permanental process to write (1.6). The expression of the Lévy measure (1.6) has been obtained in [10] by different means.

Besides (1.5) represents a new result on the local time process that can not be extended to the general case. More precisely, in the general case the processes $r^{(a)}$ and $\mathcal{L}^{(a)}$ respectively defined in (1.1) and Theorem 1.1, are different processes. Actually in general $r^{(a)}$ is not even infinitely divisible while $\mathcal{L}^{(a)}$ is always infinitely divisible. Still, we emphasize the fact that the expression (1.6) of $\mu$ is an illustration of the general result (1.2). Indeed the life time $\zeta$ of $X$ under $\tilde{P}^{a}$ is equal to $\int_{E} L_{\infty}^{(a)}(x) m(d x)$ and hence (1.6) can also be written as follows:

$$
2 \mu(F)=\int_{E} \mathbb{E}\left[\frac{F\left(L_{\infty}^{(a)}\right)}{\int_{E} L_{\infty}^{(a)}(x) m(d x)}\right] \mathbb{E}\left[\frac{1}{2} \Psi(a)\right] m(d a) .
$$

Finally note that if one assumes that $E$ is a separable metric space and $\psi$ is stochastically continuous (e.g. the function $g$ is continuous w.r.t. the metric on $E$ ), then the expression of $\mu$ given by (1.7) is still available if one replaces $m$ by any $\sigma$-finite measure $\tilde{m}$ with support equal to $E$ such that $\int_{E} \mathbb{E}[\psi(x)] \tilde{m}(d x)<\infty$.

In the special case of permanental processes, Theorem 1.3 provides the decomposition below. To introduce it we use the following notation.

Consider a continuous additive functional $\left(A_{t}\right)_{t \geq 0}$ defined by

$$
A_{t}=\int_{E} L_{t}^{x} \nu_{A}(d x)
$$

where $\nu_{A}$ is the so-called Revuz measure of $A$. We denote by $V$ the fine support of $A$. Note that $V$ is contained in the support of $\nu_{A}$.

We assume that: $\int_{V} g(x, x) \nu_{A}(d x)<\infty$.

Define $h_{t}=\inf \left\{s \geq 0: A_{s}>t\right\}$. The process $X^{V}=\left(X_{h_{t}}, t \geq 0\right)$ is a transient Markov process living on $V$. Its total accumulated local times process is $\left(L_{\infty}^{x}, x \in V\right)$ and its 0 -potential densities are $(g(x, y),(x, y) \in V \times V)$ with respect to the measure $\nu_{A}$. Denote by $\psi^{V}$ (resp. $\Psi^{V}$ ) the permanental process associated to $\left(X_{h_{t}}, t \geq 0\right)$ with index 2 (resp. 1). Since the law of a permanental process is completely determined by its kernel, one obtains:

$$
(\psi(x), x \in V) \stackrel{\text { (law) }}{=}\left(\psi^{V}(x), x \in V\right)
$$


and

$$
(\Psi(x), x \in V) \stackrel{\text { law })}{=}\left(\Psi^{V}(x), x \in V\right) .
$$

Let $T_{V}$ be the first hitting time of $V: T_{V}=\inf \left\{t>0: X_{t} \in V\right\}$, and denote by $g_{T_{V}}$ the 0 -potential densities of $X$ killed at $T_{V}$ and by $\psi_{g_{T_{V}}}$ the permanental process with index 2 and kernel $g_{T_{V}}$.

The following result can be viewed as a generalization of Theorem 1.4 which corresponds to the case where $\nu_{A}$ is the Dirac measure with unit mass at $a$.

Theorem 1.8. For any $V$ chosen as above, $\psi$ admits the following decomposition into the sum of two independent nonnegative infinitely divisible processes

$$
\psi \stackrel{\text { law) }}{=} \psi_{g_{T_{V}}}+\phi^{V},
$$

such that the Lévy measure of $\left(\phi^{V}, x \in E\right)$ is the law of $\left(L_{\infty}^{x}, x \in E\right)$ under $\frac{1}{2} \int_{V} \tilde{\mathbb{E}}_{a}\left[\frac{1}{A_{\infty}} ; \cdot\right] g(a, a) \nu_{A}(d a)$.

Moreover we have:

- the restriction to $V$ of $\psi_{g_{T}}$ is nul.

- the restriction to $V$ of $\phi^{V}$ has the law of $\psi^{V}$.

- $\psi_{g_{T_{V}}} \stackrel{(\text { law })}{=}\left(\psi \mid \int_{V} \psi(x) \nu_{A}(d x)=0\right)$.

Note that although the restriction to $V$ of $\phi^{V}$ is a permanental process, $\phi^{V}$ is not a permanental process.

Theorem 1.8 implies that the Lévy measure of $\psi_{\left.\right|_{V}}$ is the law of $\left(L_{\infty}^{x}, x \in V\right)$ under $\frac{1}{2} \int_{V} \tilde{\mathbb{E}}_{a}\left[\frac{1}{A_{\infty}} ;.\right] g(a, a) \nu_{A}(d a)$ and that the Lévy measure of $\psi_{g_{T_{V}}}$ is $\mu\left(T_{V}=\infty ;.\right)$. These two facts have been already established in [10] (Theorem 6.1, Theorem 7.3 and Corollary 7.4).

Finally in section 2, we try to give an answer to the question of the existence of Dynkin's isomorphism Theorem. Namely given the family of local time processes $\left(L_{\infty}^{(a)}, a \in E\right)$ associated to the transient Markov process $X$, which property determines the existence of a nonnegative process $\psi$, independent of $X$, satisfying the following identity in law for every $a$ in $E$ :

$$
\psi+L_{\infty}^{(a)} \text { has the law of } \psi \text { under } \mathbb{E}\left[\frac{\psi(a)}{\mathbb{E}[\psi(a)]}, .\right] \text { ? }
$$

We provide a general answer. Actually we answer the following more general question: Given a family of nonnegative processes $\left(r^{(a)}, a \in E\right)$, under what condition there exists a nonnegative process $\psi$ satisfying (1.1)? We already know (see [5], [16]) that when such a process $\psi$ exists, then it has to be infinitely divisible.

The paper is organized as follows. All the proofs of the results presented in the introduction are given in section 6 . In section 2 , we establish a converse of (1.1), the general isomorphism Theorem. Under the assumption of the continuity of the local time process, section 3 lists various properties that the associated permanental process must satisfy thanks to Theorem 1.4. Section 4 provides an expression of the Lévy measure of squared Bessel processes. Section 5 presents some remarks on Theorem 1.4.

\section{A converse to (1.1)}

Let $\left(r^{(a)} ; a \in E\right)$ be a family of nonnegative processes. It is natural to ask under what condition on the corresponding family of laws, there exists a nonnegative infinitely divisible process $\left(\psi_{x}, x \in E\right)$ without drift, satisfying (1.1). To answer this question we will make use of a necessary condition that appeared in the proof of Theorem 1.2 but also of the following characterization of Lévy measures established by Rosinski [16]. We adapt it to our framework of nonnegative infinitely divisible processes. 
Lévy measures Let $\mu$ be a measure on $\left(\mathbb{R}_{+}^{E}, \mathcal{B}^{E}\right)$, where $\mathcal{B}^{E}$ denotes the cylindrical $\sigma$-algebra associated to $\mathbb{R}_{+}^{E}$ the space of all functions from $E$ into $\mathbb{R}_{+}$. There exists an infinitely divisible nonnegative process $\left(\psi_{x}, x \in E\right)$ such that for every $n>0$, every $x_{1}, . ., x_{n}$ in $E$ :

$$
\mathbb{E}\left[\exp \left\{-\sum_{i=1}^{n} \alpha_{i} \psi_{x_{i}}\right\}\right]=\exp \left\{-\int_{\mathbb{R}_{+}^{E}}\left(1-e^{-\sum_{i=1}^{n} \alpha_{i} y\left(x_{i}\right)}\right) \nu(d y)\right\},
$$

iff $\mu$ satisfies the two following conditions:

(L1) for every $x \in E \mu(|y(x)| \wedge 1))<\infty$,

(L2) for every $A \in \mathcal{B}^{E}, \mu(A)=\mu_{*}\left(A \backslash 0_{E}\right)$, where $\mu_{*}$ is the inner measure.

A measure $\mu$ on $\left(\mathbb{R}_{+}^{E}, \mathcal{B}^{E}\right)$, is said to be a Lévy measure if it satisfies (L1) and (L2),

Conversely to every nonnegative infinitely divisible process $\left(\psi_{x}, x \in E\right)$ with 0 -drift, corresponds a unique Lévy measure $\mu$ such that (2.1) is satisfied.

The proof of Theorem 1.2 (given in section 6) shows that the existence of a process $\psi$ satisfying (1.1) requires at least two properties from $\left(r^{(a)}, a \in E\right)$. First, for every $a$, one must have (6.4): $\mathbb{P}\left[r_{a}^{(a)}=0\right]=0$. But note also that using twice (6.5), for $a$ and $b$ any couple of points of $E$, leads to

$$
\mathbb{E}[\psi(a)] \mathbb{E}\left[r^{(a)}(b) F\left(r^{(a)}\right)\right]=\mathbb{E}[\psi(b)] \mathbb{E}\left[r^{(b)}(a) F\left(r^{(b)}\right)\right],
$$

for any measurable functional $F$.

Indeed, starting from (6.5) one has:

$$
\begin{aligned}
\mu\left(F(y) 1_{\{y(a)>0, y(b)>0\}}\right) & =\mathbb{E}[\psi(a)] \mathbb{E}\left[\frac{1}{r_{a}^{(a)}} ; F\left(r^{(a)}\right) 1_{\left\{r_{b}^{(a)}>0\right\}}\right] \\
& =\mathbb{E}[\psi(b)] \mathbb{E}\left[\frac{1}{r_{b}^{(b)}} ; F\left(r^{(b)}\right) 1_{\left\{r_{a}^{(b)}>0\right\}}\right] .
\end{aligned}
$$

Then one chooses $F(y)=y(a) y(b) \tilde{F}(y)$, to obtain (2.2).

The result below shows that if one assumes the existence of an appropriate measure $m$ on $E,(2.2)$ is also sufficient.

Theorem 2.1. Assume that $E$ is a separable metric space. Let $\left(r^{(a)} ; a \in E\right)$ be a family of nonnegative processes such that for every $a$ in $E, r^{(a)}$ is stochastically continuous. Assume that there exists a family $\left(c_{a}, a \in E\right)$ of strictly positive numbers such that for every measurable functional $F$ and every $a, b$ in $E$, we have for every $a$ in $E$ :

$$
c_{a} \mathbb{E}\left[r_{b}^{(a)} F\left(r^{(a)}\right)\right]=c_{b} \mathbb{E}\left[r_{a}^{(b)} F\left(r^{(b)}\right)\right],
$$

and there exists a $\sigma$-finite measure $m$ with support equal to $E$ such that for every $a$ in $E$ :

$$
\mathbb{P}\left[0<\int_{E} r^{(a)}(x) m(d x)<\infty\right]=1 .
$$

Then there exists a nonnegative infinitely divisible process $\psi$ with 0-drift, independent of the family $\left(r^{(a)}, a \in E\right)$, such that for every $a$ in $E$

$$
\psi+r^{(a)} \stackrel{(\text { law })}{=} \psi \text { under } \mathbb{E}\left[\frac{\psi(a)}{c_{a}} ; .\right]
$$

with a Lévy measure $\mu$ given by:

$$
\mu(F)=\int_{E} \mathbb{E}\left[\frac{F\left(r^{(a)}\right)}{\int_{E} r^{(a)}(x) m(d x)}\right] c_{a} m(d a) .
$$

Moreover if $\int_{E} c_{a} m(d a)<\infty$, then $\psi$ satisfies: $\left(\psi \mid \int_{E} \psi(x) m(d x)=0\right)=0$. 
Infinitely divisible processes

Note that (2.4) together with (2.3), imply (6.4). To see this, one writes:

$$
c_{a} \mathbb{E}\left[r_{b}^{(a)} 1_{\left\{r_{a}^{(a)}=0\right\}}\right]=c_{b} \mathbb{E}\left[r_{a}^{(b)} 1_{\left\{r_{a}^{(b)}=0\right\}}\right]=0 .
$$

Then integrate each member of the above equation with respect to $m(d b)$ to obtain: $c_{a} \mathbb{E}\left[1_{\left\{r_{a}^{(a)}=0\right\}} \int_{E} r^{(a)}(x)\right] m(d x)=0$, which leads to (6.4).

Proof. We first show that the measure $\mu$ defined by

$$
\mu(F)=\int_{E} \mathbb{E}\left[\frac{F\left(r^{(a)}\right)}{\int_{E} r^{(a)}(x) m(d x)}\right] c_{a} m(d a),
$$

is a Lévy measure on $\left(\mathbb{R}_{+}^{E}, \mathcal{B}^{E}\right)$. We show that $\mu$ satisfies the two conditions (L1) and (L2).

For every $b$ in $E$, we have:

$$
\begin{aligned}
c_{b} \mathbb{E}\left[F\left(r^{(b)}\right)\right] & =c_{b} \mathbb{E}\left[F\left(r^{(b)}\right) \frac{\int_{E} r^{(b)}(x) m(d x)}{\int_{E} r^{(b)}(x) m(d x)}\right] \\
& =\int_{E} c_{b} \mathbb{E}\left[\frac{r^{(b)}(a) F\left(r^{(b)}\right)}{\int_{E} r^{(b)}(x) m(d x)}\right] m(d a) \\
& =\int_{E} c_{a} \mathbb{E}\left[\frac{r^{(a)}(b) F\left(r^{(a)}\right)}{\int_{E} r^{(a)}(x) m(d x)}\right] m(d a) .
\end{aligned}
$$

Hence one obtains in particular (for $F=1$ )

$$
c_{b}=\int_{E} c_{a} \mathbb{E}\left[\frac{r^{(a)}(b)}{\int_{E} r^{(a)}(x) m(d x)}\right] m(d a) .
$$

Consequently $\mu(|y(b)|)<\infty$ and $\mu$ satisfies (L1).

To show that $\mu$ satisfies (L2), it is sufficient to show that there exists a countable subset $T$ of $E$ such that $\mu\left(\left\{y \in \mathbb{R}_{+}^{E}: y_{\left.\right|_{T}}=0\right\}\right)=0$ (see [16] Remark 2.2).

Let $D$ be a countable dense subset of $E$. Since, for every $a$ in $A, r^{(a)}$ is stochastically continuous, $D$ can be used as separability set for $r^{(a)}$. Hence:

$$
1_{\left\{r_{x}^{(a)}=0, \forall x \in D\right\}} \leq 1_{\left\{r_{x}^{(a)}=0, \forall x \in D \cap B(a, \varepsilon)\right\}} \leq 1_{\left\{r_{a}^{(a)}=0\right\}}=0 \text { a.s. }
$$

which leads to: $\mu\left(0_{\left.\right|_{D}}\right)=0$. Consequently $\mu$ satisfies (L2).

Denote by $\left(\psi_{x}, x \in E\right)$ a nonnegative infinitely divisible process with Lévy measure $\mu$ and no drift. For every $x_{1}, x_{2}, \ldots, x_{n}$ in $E$, we have:

$$
\mathbb{E}\left[\exp \left\{-\sum_{i=1}^{n} \alpha_{i} \psi_{x_{i}}\right\}\right]=\exp \left\{-\int_{E} c_{a} \mathbb{E}\left[\frac{1-e^{-\sum_{i=1}^{n} \alpha_{i} r^{(a)}\left(x_{i}\right)}}{\int_{E} r^{(a)}(x) m(d x)}\right] m(d a)\right\}
$$

hence, if one sets $b=x_{1}$

$$
\mathbb{E}\left[\psi_{b} \exp \left\{-\sum_{i=1}^{n} \alpha_{i} \psi_{x_{i}}\right\}\right]=\int_{E} c_{a} \mathbb{E}\left[r^{(a)}(b) \frac{e^{-\sum_{i=1}^{n} \alpha_{i} r^{(a)}\left(x_{i}\right)}}{\int_{E} r^{(a)}(x) m(d x)}\right] \mathbb{E}\left[\exp \left\{-\sum_{i=1}^{n} \alpha_{i} \psi_{x_{i}}\right\}\right] m(d a) .
$$

One notes that using (2.6), one has

$$
\int_{E} c_{a} \mathbb{E}\left[\frac{r^{(a)}(b) e^{-\sum_{i=1}^{n} \alpha_{i} r^{(a)}\left(x_{i}\right)}}{\int_{E} r^{(a)}(x) m(d x)}\right] m(d a)=c_{b} \mathbb{E}\left[\exp \left\{-\sum_{i=1}^{n} \alpha_{i} r^{(b)}\left(x_{i}\right)\right\}\right],
$$

which with (2.7) leads to (1.1).

If $\int_{E} c_{a} m(d a)<\infty$, using Theorem 1.3 for $V=E$, one knows that the Lévy measure of $\mathcal{L}^{V}$ is precisely (2.5). Hence it coincides with $\psi$ and one obtains: $\left(\psi \mid \int_{E} \psi(x) m(d x)=\right.$ $0)=0$ a.s. 
Remark 2.2. Theorem 1.2 provides the explicit expression (1.2) of the Lévy measure of stochastically continuous nonnegative infinitely divisible processes $\psi$ without drift. As a consequence of this result one obtains that for every measurable functional $F$ on $\mathbb{R}_{+}^{E}$, the quantity $\int_{E} \mathbb{E}[\psi(a)] \mathbb{E}\left[\frac{F\left(r^{(a)}\right)}{\int_{E} r^{(a)}(x) m(d x)}\right] m(d a)$ is independent of the choice of the measure $m$. We show now that this remarkable property can be seen as a consequence of (2.2). We assume that for any $a$ in $E, \mathbb{E}[\psi(a)]>0$.

For any measure $m$ satisfying the assumptions of Theorem 1.2 , define a measure $J_{m}$ by:

$$
J_{m}(F)=\int_{E} \mathbb{E}[\psi(a)] \mathbb{E}\left[\frac{F\left(r^{(a)}\right)}{\int_{E} r^{(a)}(x) m(d x)}\right] m(d a) .
$$

We show that: $J_{m}(F)=J_{\tilde{m}}(F)$, for any other measure $\tilde{m}$ satisfying the assumptions of Theorem 1.2.

(1) Note that if $F$ is such that: $F(y)=y(a) \tilde{F}(y)$ for some $a$ in $E$, then similarly as for (2.6), using (2.2) one has: $J_{m}(F)=J_{\tilde{m}}(F)$.

(2) One easily shows that if $\psi$ is stochastically continuous, then for every $a$ in $E$ such that $\mathbb{E}[\psi(a)]>0, r^{(a)}$ is stochastically continuous too. Let $D$ be a countable dense subset of $E, D=\left\{a_{n}, n \geq 1\right\}$. For every $a$ in $E, D$ can be chosen as separability set for $r^{(a)}$. Hence one obtains: $J_{m}\left(\left\{y: y_{\left.\right|_{D}}=0\right\}\right)=0$.

Consequently: $J_{m}(F)=\sum_{k=1}^{\infty} J_{m}\left(F, B_{k}\right)$, where $B_{1}=\left\{y \in \mathbb{R}_{+}^{E}: y\left(a_{1}\right)>0\right\}$ and for $k \geq 2, B_{k}=\left\{y \in \mathbb{R}_{+}^{E}: y\left(a_{k}\right)>0, y\left(a_{j}\right)=0 ; 1 \leq j \leq k-1\right\}$.

One has: $F(y) 1_{B_{k}}(y)=y\left(a_{k}\right)\left(\frac{F(y)}{y\left(a_{k}\right)} 1_{y\left(a_{k}\right)>0} 1_{B_{k}}(y)\right)$. Hence thanks to (1), for every $k \geq 1: J_{m}\left(F, B_{k}\right)=J_{\tilde{m}}\left(F, B_{k}\right)$, which finally leads to: $J_{m}(F)=J_{\tilde{m}}(F)$.

\section{Trajectorial properties of the permanental process}

In this section we consider permanental processes admitting for kernel the 0-potential densities of a transient Markov process with a locally compact metric state space. We always assume that the local time process of this transient Markov process is continuous as a process indexed by time and space.

\subsection{0-1 laws}

We assume that $E$ is a compact separable metric space. Let $D$ be a dense subset of $E, D=\left\{a_{n}, n \geq 1\right\}$.

First note that the continuity of the local time implies the continuity of $g$ and of $g_{T_{a}}$. (For example, since $\lim _{b \rightarrow a} \mathbb{P}_{a}\left[L_{\infty}^{b}>0\right]=1$, it follows that: $\lim _{b \rightarrow a} g(a, b)=g(a, a)$ ).

Since: $\left.\mathbb{E}\left[\left(\frac{\Psi_{a}}{2}-\frac{\Psi_{b}}{2}\right)^{2}\right]=3(g(a, a))^{2}+3(g(b, b))^{2}-2 g(a, a) g(b, b)-4 g(a, b) g(b, a)\right)$, it follows that he continuity of the kernel $g$ is equivalent to the $L^{2}$-continuity of $\Psi$.

We have, using Theorem 1.4

$$
\Psi \stackrel{\text { (law) }}{=} \Psi_{g_{T_{a_{1}}}}+2 L^{\left(a_{1}\right)}
$$

Similarly

$$
\Psi_{g_{T_{1}}} \stackrel{\text { (law) }}{=} \Psi_{g_{T_{a_{1}} \wedge T_{a_{2}}}}+2 L^{\left(a_{1}, a_{2}\right)}
$$

where $\Psi_{g_{T_{1} \wedge T_{a_{2}}}}$ is a permanental process independent of $X$, with kernel the potential density of $X$ killed at $T_{a_{1}} \wedge T_{a_{2}}$, and index 1 and $L^{\left(a_{1}, a_{2}\right)}$ has the law of the local time process of $X$ killed at $T_{a_{1}}$, conditioned to start at $a_{2}$ and killed at its last visit to $a_{2}$.

Hence

$$
\Psi \stackrel{\text { (law) }}{=} \Psi_{g_{T_{a_{1}} \wedge T_{a_{2}}}}+2 L^{\left(a_{1}\right)}+2 L^{\left(a_{1}, a_{2}\right)}
$$

with the three terms on the right hand side independent. 
Infinitely divisible processes

Iterating the procedure, one obtains $\Psi$ as the sum of $n+1$ independent terms as follows:

$$
\Psi^{(\text {law })} \stackrel{{ }^{\prime}}{=} \varphi_{n}+2 L^{\left(a_{1}\right)}+2 L^{\left(a_{1}, a_{2}\right)}+\ldots+2 L^{\left(a_{1}, a_{2}, \ldots, a_{n}\right)}
$$

with $\varphi_{n}=\Psi_{g_{T_{1} \wedge T_{a_{2}} \wedge \ldots \wedge T_{a_{n}}}}$, and for every $2 \leq k \leq n, L^{\left(a_{1}, a_{2}, \ldots, a_{k}\right)}$ has the law of the local time process of $X$ conditionned to start at $a_{k}$ and killed at the last exit from $a_{k}$ before $T_{a_{1}} \wedge T_{a_{2}} \wedge \ldots \wedge T_{a_{k-1}}$.

Except for $\varphi_{n}$, the $n+1$ terms on the right hand side of (3.1) are continuous on $E$.

For simplicity, we just write $L(k)$ instead of $L^{\left(a_{1}, a_{2}, \ldots, a_{k}\right)}$ for $k \geq 1$. Hence for every $n$, one has:

$$
\frac{1}{2} \Psi \stackrel{(\text { law })}{=} \frac{1}{2} \varphi_{n}+\sum_{k=1}^{n} L(k)
$$

We show now that there exists a finite nonnegative process $(Y(x), x \in E)$ independent of $X$ such that for every $x: Y(x)=0$ a.s., and

$$
\Psi \stackrel{(\text { law })}{=} Y+2 \sum_{k=1}^{\infty} L(k) .
$$

Note that the sequence of processes $\left(\varphi_{n}\right)$ is stochastically decreasing and bounded below by 0 . Hence there exists a sequence of nonnegative processes $\left(Y_{n}\right)_{n \geq 0}$ defined on the same space such that for every $n: Y_{n} \stackrel{(\text { law })}{=} \varphi_{n}$, and for every $n$

$$
0 \leq Y_{n+1}(x) \leq Y_{n}(x)
$$

For a fixed $\omega,\left(Y_{n}(x), n \geq 1\right)$ decreases to some value that we denote by $Y(x)\left(Y_{n}\right.$ converges pointwise to $Y$ ). Since the local time is assumed to be continuous, in particular $L_{T_{D}}$ is continuous, where $T_{D}=\inf \left\{t \geq 0: X_{t} \in D\right\}$. But $L_{T_{D}}=0$ on $D$, hence $L_{T_{D}}=0$ on $E$ and $g_{T_{D}}=0$ on $E \times E$. Consequently for every $x$ in $E, \mathbb{E}\left[Y_{n}(x)\right]$ decreases to 0 . One obtains: $\mathbb{E}[Y(x)]=0$ and hence $Y(x)=0$ a.s.

On the other hand the sequence of processes $\left(\sum_{k=1}^{n} L(k)\right)_{n \geq 1}$ is increasing. For a fixed $\omega, \sum_{k=1}^{n} L(k)(x)$ increases to $\sum_{k=1}^{\infty} L(k)(x)$ (a value that might be infinite).

For every $n$, set:

$$
\frac{1}{2} \psi_{n}=\frac{1}{2} Y_{n}+\sum_{k=1}^{n} L(k) .
$$

Consequently, for a fixed $\omega, \psi_{n}(x)$ converges to some value $\psi_{\infty}(x)$. The process $\psi_{\infty}$ satisfies:

$$
\frac{1}{2} \psi_{\infty}=\frac{1}{2} Y+\sum_{k=1}^{\infty} L(k) .
$$

For every $n$, for every $x_{1}, x_{2}, \ldots, x_{p}$ in $E$ the vectors $\left(\psi_{n}\left(x_{1}\right), \psi_{n}\left(x_{2}\right), \ldots \psi_{n}\left(x_{p}\right)\right)$ all live in the same probability space and have the law of $\left(\Psi\left(x_{1}\right), \Psi\left(x_{2}\right), \ldots, \Psi\left(x_{p}\right)\right)$. Consequently:

$$
\left(\psi_{\infty}\left(x_{1}\right), \psi_{\infty}\left(x_{2}\right), \ldots \psi_{\infty}\left(x_{p}\right)\right) \stackrel{(\text { law })}{=}\left(\Psi\left(x_{1}\right), \Psi\left(x_{2}\right), \ldots \Psi\left(x_{p}\right)\right)
$$

which leads to (3.2).

Since $Y$ admits a version which identically equals 0 , one obtains that $2 \sum_{k=1}^{\infty} L(k)$ is a version of $\Psi$. Another consequence is:

$$
\mathbb{P}\left[\forall x \in E: \sum_{k=1}^{\infty} L(k)(x)<\infty\right]=1 .
$$


Define the oscillation function of a random separable process $Z(x)_{x \in E}$ by

$$
\operatorname{osc}_{Z}(a)=\lim _{\delta \rightarrow 0} \sup _{u, v \in B(a, \delta)}|Z(u)-Z(v)| .
$$

As a consequence of (3.2), one obtains for any separable version of $\Psi$ (that we still denote by $\Psi$ ) that there exists an upper semi-continuous deterministic function $w$ such that

$$
\mathbb{P}\left[\operatorname{osc}_{\Psi}(a)=w(a), \forall a \in E\right]=1
$$

As an immediate consequence, $\Psi$ enjoys many $0-1$-laws. For example,

$$
\begin{gathered}
\mathbb{P}[\Psi \text { is continuous on } E]=0 \text { or } 1 \\
\mathbb{P}\left[\lim _{y \longrightarrow a} \Psi(y)=\Psi(a)\right]=0 \text { or } 1
\end{gathered}
$$

$\mathbb{P}[\Psi$ has a bounded discontinuity on $E]=0$ or 1

$\mathbb{P}[\Psi$ has a bounded discontinuity at $a]=0$ or 1

$$
\begin{gathered}
\mathbb{P}[\Psi \text { is unbounded on } E]=0 \text { or } 1 \\
\mathbb{P}\left[\limsup _{x \longrightarrow a} \Psi(x)=+\infty\right]=0 \text { or } 1
\end{gathered}
$$

Note that the processes $\varphi_{n}, n \geq 1$, enjoy the same properties simultaneously and have the same oscillation function $w$.

By Fatou's Lemma, one has: $\mathbb{E}\left[\liminf _{x \longrightarrow a_{1}} \varphi_{1}(x)\right] \leq \liminf _{x \longrightarrow a_{1}} \mathbb{E}\left[\varphi_{1}(x)\right]=0$. Hence: $\mathbb{P}\left[\liminf \operatorname{in}_{x \longrightarrow a_{1}} \varphi_{1}(x)=0\right]=1$, which leads to

$$
\mathbb{P}\left[\liminf _{x \longrightarrow a_{1}} \Psi(x)=\Psi\left(a_{1}\right)\right]=1
$$

Since the choice of $a_{1}$ is abitrary one finally obtains:

$$
\mathbb{P}\left[\liminf _{x \longrightarrow a} \Psi(x)=\Psi(a)\right]=1, \forall a \in E
$$

and hence to

$$
\lim _{\delta \longrightarrow 0} \sup _{x \in B(a, \delta)} \Psi(x)=\limsup _{x \longrightarrow a} \Psi(x)=\Psi(a)+w(a) \text { a.s. }
$$

Using (3.3) and Theorem 7 p.213 in [17], for every fixed $\omega$, there exists a dense subset $B(\omega)$ in $E$ such that $\sum_{k=1}^{\infty} L(k)(\omega)$ is continuous at each point of $B(\omega)$.

Now, the set $A=\{x \in E: w(x)=0\}$ is a deterministic set and contains $B(\omega)$ for every $\omega$. Hence: $\bar{A}=E$, and at least $A$ is dense in $E$. Since $A$ is deterministic, it contains a deterministic dense set $\Delta$. One hence obtains the following result.

Proposition 3.1. Assume that $X$ is a transient Markov process with a continuous local time process then there exists a dense subset $\Delta$ of its state space such that its associated permanental process $\psi$ is continuous at each point of $\Delta$ and $\psi_{\left.\right|_{\Delta}}$ is continuous.

The following proposition has been already obtained by Marcus and Rosen. Their argument is based on Barlow's necessary and sufficient condition for a Lévy process to have a continuous local time process [1] and on a sufficient condition for a permanental process to be continuous [13]. Our proof has the merit to be a direct argument relying exclusively on Theorem 1.4 .

Proposition 3.2. Assume that $X$ is a transient Lévy process with continuous local time process. Then its associated permanental process admits a continuous version.

Proof. Indeed in this case the oscillation function of the associated permanental is equal to a constant function. Thanks to Proposition 3.1, we know that it is equal to 0 on a dense set, and hence the oscillation function is equal to 0 at each point. 


\subsection{The special symmetric case}

In case the transient Markov process $X$ admits a symmetric potential density w.r.t. a reference measure $m$, then the permanental process is a squared Gaussian process. Namely, $(\psi(x), x \in E)=\left(\eta^{2}(x), x \in E\right)$ with $(\eta(x), x \in E)$ centered Gaussian process with covariance $g$. The process $(\eta(x), x \in E)$ is the so-called associated Gaussian process to $X$. The following proposition has already been obtained with other arguments in [14], but appears as an immediate consequence of section 3.1.

Proposition 3.3. Let $X$ be a transient symmetric Markov process with a continuous local time process. Denote by $\eta$ its associated Gaussian process. Then for any point $a$ in $E$, if $\eta$ is not unbounded at a then $\eta$ is continuous at $a$.

Indeed, we know (see [11]) that there exists a deterministic function $\delta$ such that

$$
\limsup _{x \longrightarrow a} \eta^{2}(x)=\left(\eta(a)+\frac{\delta(a)}{2}\right)^{2} .
$$

But the assumption of continuity of the local time process, gives the existence of a deterministic function $w_{1}$ such that:

$$
\limsup _{x \longrightarrow a} \eta^{2}(x)=\eta^{2}(a)+w_{1}(a) .
$$

If $w_{1}(a)<\infty$, then immediately one has: $\delta(a)=w_{1}(a)=0$.

\subsection{Real indexed permanental processes}

In case the transient Markov process $X$ is real valued, then its associated permanental process is indexed by $\mathbb{R}$.

Proposition 3.4. Let $X$ be a real valued transient Markov process with a continuous local time process. If its associated permanental process $\Psi$ has càdlàg trajectories then $\Psi$ has a continuous version.

Proof. Fix $a$ in R. We assume that $\lim _{x \rightarrow a, x>a} \Psi(x)=\Psi(a)$ and $\lim _{x \rightarrow a, x<a} \Psi(x)=\Psi_{-}(a)$. We have established in section 3.1 that:

$$
\limsup _{x \longrightarrow a} \Psi(x)=\Psi(a)+w(a) \text { a.s. }
$$

and

$$
\liminf _{x \rightarrow a} \Psi(x)=\Psi(a) \text { a.s. }
$$

Consequently, one obtains: $w(a)<\infty$ and $\Psi_{-}(a)=\Psi(a)+w(a)$ a.s.

Let $\left(x_{n}\right)$ be a strictly increasing sequence converging to $a$.

On one hand, one has: $\Psi\left(x_{n}\right) \rightarrow_{n \rightarrow \infty} \Psi(a)+w(a)$ a.s. But on the other hand, one has: $\Psi\left(x_{n}\right) \rightarrow_{n \rightarrow \infty} \Psi(a)$ in $L^{2}$, which implies that there exists a subsequence $\left(x_{f(n)}\right)$ of $\left(x_{n}\right)$ such that $\Psi\left(x_{f(n)}\right) \rightarrow_{n \rightarrow \infty} \Psi(a)$ a.s.

One hence obtains: $w(a)=0$.

\section{Lévy measure of squared Bessel processes}

Given a nonnegative infinitely divisible process $\psi$ without drift, to obtain its Lévy measure one needs only to identify for any $a$ such that $\mathbb{E}\left[\psi_{a}\right]>0$, the law of $r^{(a)}$ (defined by (1.1)). To answer a question asked by Zhan Shi and Jan Rosinski, we detail the use of Theorem 1.2 in the special case of squared Bessel processes. Let $\left(\psi_{t}, t \geq 0\right)$ be a squared Bessel process with dimension $\delta$ starting from $x$. Denote its Lévy measure by $\mu^{\delta, x}$. The additivity property of squared Bessel processes immediately gives:

$$
\mu^{\delta, x}=\delta \mu^{1,0}+x \mu^{0,1} .
$$


It is hence sufficient to compute $\mu^{1,0}$ and $\mu^{0,1}$ to obtain the following proposition.

Proposition 4.1. The Lévy measure $\mu^{\delta, x}$ of a squared Bessel process with dimension $\delta$ starting from $x$ is given by

$$
\mu^{\delta, x}(F)=\int_{0}^{\infty} \int_{0}^{\infty} e^{-a}\left(\delta+x \frac{\ell}{2 a}\right) \frac{e^{-\frac{\ell}{2 a}}}{2 a} \mathbb{E}\left[\frac{F\left(X^{\ell, a}\right)}{\int_{0}^{\infty} X_{t}^{\ell, a} e^{-t} d t}\right] d a d \ell
$$

for every measurable functional $F$ on $\mathbb{R}_{+}^{\mathbb{R}_{+}}$, where the process $\left(X_{t}^{\ell, a}, t \geq 0\right)$ is a nonnegative Markov process such that: $X_{a}^{\ell, a}=\ell,\left(X_{t+a}^{\ell, a}, t \geq 0\right)$ is a squared Bessel process with dimension 0 starting from $\ell$ and $\left(X_{a-t}^{\ell, a}, 0 \leq t \leq a\right)$ is a squared Bessel bridge with dimension 0 , lenght $a$, between $\ell$ and 0 .

Proof. We show below how to obtain the law of $r^{(a)}$ in the case of a squared Bessel process with dimension 1 starting from 0 and in the case of dimension 0 starting from 1 . We could use the famous Ray-Knight Theorems for that, but in both cases it is shorter to just use the definition of $r^{(a)}$.

Let $\left(\psi_{t}, t \geq 0\right)$ be a squared Bessel process starting from 0 with dimension 1 . For every $a>0, \mathbb{E}\left[\psi_{a}\right]=a$. To compute the law of $r^{(a)}$, we write:

$$
\psi+r^{(a) \stackrel{(\text { law })}{=}} \psi \text { under } \mathbb{E}\left[\frac{\psi(a)}{a}, .\right]
$$

First we note that:

$$
\mathbb{E}\left[e^{-\lambda \psi_{a}}\right] \mathbb{E}\left[e^{-\lambda r_{a}^{(a)}}\right]=\mathbb{E}\left[\frac{\psi_{a}}{a} e^{-\lambda \psi_{a}}\right]
$$

Since: $\mathbb{E}\left[e^{-\lambda \psi_{a}}\right]=(1+2 \lambda a)^{-1 / 2}$, (4.2) shows that $r_{a}^{(a)}$ has an exponential law with parameter $\frac{1}{2 a}$.

For any $x_{1}, x_{2}, \ldots, x_{n}$ in $[0, \infty)$ and $\lambda_{1}, \ldots, \lambda_{n}$ in $\mathbb{R}_{+}$, we have, making use of the Markov property of $\psi$ with obvious notation:

$$
\begin{aligned}
\mathbb{E}\left[\exp \left\{-\sum_{k=1}^{n} \lambda_{k} \psi_{x_{k}+a}\right\}\right] \mathbb{E}\left[\exp \left\{-\sum_{k=1}^{n} \lambda_{k} r_{x_{k}+a}^{(a)}\right\}\right] & =\frac{1}{a} \mathbb{E}\left[\psi_{a} \exp \left\{-\sum_{k=1}^{n} \lambda_{k} \psi_{x_{k}+a}\right\}\right] \\
& =\frac{1}{a} \mathbb{E}\left[\psi_{a} \mathbb{E}\left[\exp \left\{-\sum_{k=1}^{n} \lambda_{k} \psi_{x_{k}}\right\} \circ \theta_{a} \mid \mathcal{F}_{a}\right]\right] \\
& =\frac{1}{a} \mathbb{E}\left[\psi_{a} \mathbb{E}_{\psi_{a}}\left[\exp \left\{-\sum_{k=1}^{n} \lambda_{k} \psi_{x_{k}}\right\}\right]\right]
\end{aligned}
$$

Hence $\left(r_{t+a}^{(a)}, t \geq 0\right)$ is a squared Bessel process with dimension 0 starting from an exponential law with parameter $\frac{1}{2 a}$.

For any $x_{1}, . ., x_{n}$ in $(0, a)$ and $\lambda_{1}, \ldots, \lambda_{n}$ in $\mathbb{R}_{+}$, we have:

$$
\mathbb{E}\left[\exp \left\{-\sum_{k=1}^{n} \lambda_{k} \psi_{a-x_{k}}\right\}\right] \mathbb{E}\left[\exp \left\{-\sum_{k=1}^{n} \lambda_{k} r_{a-x_{k}}^{(a)}\right\}\right]=\frac{1}{a} \mathbb{E}\left[\psi_{a} \exp \left\{-\sum_{k=1}^{n} \lambda_{k} \psi_{a-x_{k}}\right\}\right],
$$

which shows that $\left(r_{a-t}^{(a)}, 0 \leq t \leq a\right)$ has the law of a squared Bessel bridge with dimension 0 and length $a$, starting with an exponential law with parameter $\frac{1}{2 a}$ and ending at 0 .

One shows (e.g. similarly as in [3]) that $r^{(a)}$ has the Markov property, hence conditionally to $r_{a}^{(a)},\left(r_{a-t}^{(a)}, 0 \leq t \leq a\right)$ and $\left(r_{t+a}^{(a)}, t \geq 0\right)$ are independent. The law of $r^{(a)}$ is hence fully described. Finally we have to choose a measure $m$ such that: $\int_{0}^{\infty} a m(d a)<\infty$. One can choose: $m(d a)=e^{-a} d a$.

A similar computation gives the law of $r^{(a)}$ when $\psi$ is a squared Bessel process with dimension 0 starting from 1 . In this case one obtains: 
- For every $a, \mathbb{E}\left[\psi_{a}\right]=1$.

- $r_{a}^{(a)}$ has a gamma law $\Gamma(2,2 a)$.

- Conditionnally to $r_{a}^{(a)}=\ell,\left(r_{t+a}^{(a)}, t \geq 0\right)$ has the law of a squared Bessel process with dimension 0 starting from $\ell$.

- Conditionnally to $r_{a}^{(a)}=\ell,\left(r_{a-t}^{(a)}, 0 \leq t \leq a\right)$ has the law of a squared Bessel bridge with dimension 0 starting from $\ell$ and ending at 0 .

We can also choose $m(d a)=e^{-a} d a$.

One obtains the final expression of $\mu^{\delta, x}$ thanks to (4.1).

Pitman and Yor have devoted section 4 of [15] to the description of $\mu^{\delta, x}$ in terms of the Itô excursion law of the reflecting Brownian motion.

\section{Some remarks on Theorem 1.4}

\subsection{Some applications}

Let $X$ be a recurrent Markov process with state space $E$, admitting a local time process $\left(L_{t}^{x}, x \in E, t \geq 0\right)$. For every $r \geq 0$, set: $\tau_{r}=\inf \left\{t>0: L_{t}^{a}>r\right\}$ Then $X$ killed at time $\tau_{S_{\theta}}$ with $S_{\theta}$ an independent exponential variable with parameter $\theta$, is transient. Theorem 1.4 leads to:

$$
\frac{1}{2} \Psi_{g_{\tau_{S_{\theta}}}} \stackrel{\text { (law) }}{=} \frac{1}{2} \Psi_{g_{T_{a}}}+L_{\tau_{S_{\theta}}}^{(a)}
$$

where $g_{\tau_{S_{\theta}}}$ denotes the 0-potential densities of $X$ killed at $\tau_{S_{\theta}}$ and $g_{T_{a}}$ the 0-potential densities of $X$ killed at $T_{a}$ its first hitting time of $a$.

The above identity leads to:

$$
\frac{1}{2}\left(\Psi_{g_{\tau_{S_{\theta}}}} \mid \Psi_{g_{\tau_{S_{\theta}}}}(a)=r\right) \stackrel{(\text { law })}{=} \frac{1}{2} \Psi_{g_{T_{a}}}+L_{\tau_{r}}^{(a)}
$$

and to

$$
\left(\Psi_{g_{\tau_{S_{\theta}}}} \mid \Psi_{g_{\tau_{S_{\theta}}}}(a)=0\right) \stackrel{(\text { law })}{=} \Psi_{g_{T_{a}}} .
$$

Denote by $\psi$ and $\tilde{\psi}$ two independent permanental processes with kernel $g_{\tau_{S_{\theta}}}$ and index 2 . This implies that:

$$
\Psi_{g_{\tau_{\theta}}} \stackrel{(\text { law })}{=} \psi+\tilde{\psi}
$$

We use now a remarkable property of permanental processes that has been noticed in [7] (Remark 2.5.1) for every $p, q \geq 0$ such that $p+q=r$

$$
(\psi \mid \psi(a)=p)+(\tilde{\psi} \mid \tilde{\psi}(a)=q) \stackrel{(\text { law })}{=}(\psi \mid \psi(a)=r)+(\tilde{\psi} \mid \tilde{\psi}(a)=0) .
$$

One hence obtains:

$$
\left(\Psi_{g_{\tau_{S_{\theta}}}} \mid \Psi_{g_{\tau_{S_{\theta}}}}(a)=r\right) \stackrel{(\text { law })}{=}(\psi \mid \psi(a)=r)+(\tilde{\psi} \mid \tilde{\psi}(a)=0)
$$

to conclude that $\mathbb{P}_{a}$ a.s.

$$
\frac{1}{2}(\psi \mid \psi(a)=r) \stackrel{(\text { law })}{=} \frac{1}{2}(\psi \mid \psi(a)=0)+L_{\tau_{r}}^{(a)},
$$

which means that $\mathbb{P}_{a}$ a.s.

$$
\frac{1}{2}(\psi \mid \psi(a)=r) \stackrel{(\text { law })}{=} \frac{1}{2} \psi_{g_{T_{a}}}+L_{\tau_{r}}^{(a)} .
$$

This last identity has been established in [7] extending a previous result of [8]. These identities in law are called isomorphism theorems because they can be seen as variants of 
the seminal Dynkin's isomorphism Theorem [2]. Theorem 1.4 represents a more general identity than (5.1) and can also generate new identities that are not obvious starting from the known isomorphism theorems. For example, assume that $X$ is a recurrent Markov process killed at an independent exponential time $S$. Denote by $g_{S}$ its 0-potential densities then for every $a$ in $E$, we have, using Theorem 1.4:

$$
\frac{1}{2} \Psi_{g_{S}} \stackrel{\text { (law) }}{=} \frac{1}{2} \Psi_{g_{T_{a} \wedge S}}+L_{S}^{(a)}
$$

or equivalently:

$$
\frac{1}{2} \Psi_{g_{S}} \stackrel{(\text { law })}{=} \frac{1}{2}\left(\Psi_{g_{S}} \mid \Psi_{g_{S}}(a)=0\right)+L_{S}^{(a)}
$$

\subsection{Selfdecomposition property}

Theorem 1.4 can be seen as an extension of the following identity for exponential variables. Denote by $\mathbf{e}_{\theta}$ an exponential variable with parameter $\theta$. Then we have for every $\lambda$ in $[0,1]$ :

$$
\mathbf{e}_{\theta} \stackrel{(\text { law })}{=} \lambda \mathbf{e}_{\theta}+X_{\lambda, \theta}
$$

where $X_{\lambda, \theta}$ is a real variable, independent of $\mathrm{e}_{\theta}$ such that

$$
\operatorname{Law}\left(X_{\lambda, \theta}\right)=(1-\lambda) \operatorname{Law}\left(\mathbf{e}_{\theta}\right)+\lambda \delta_{0} .
$$

(5.2) is a translation of the selfdecomposition property of the exponential law. It can also be seen as a characterization of the exponential law.

Each of the one dimensional identities in law implied by (1.4) is an illustration of (5.2), but (1.4) does not lead to the selfdecomposability of the permanental process since the factor $\lambda$ varies with the index $x$ in $E$.

As it has been noticed in [6], permanental vectors are not selfdecomposable, but Theorem 1.4 is reminiscent of that property.

\section{Proofs of Theorem 1.1, Theorem 1.2, Theorem 1.3, Theorem 1.4, Theorem 1.5 and Theorem 1.8}

Proof of Theorem 1.1. For $x_{1}, x_{2}, \ldots, x_{n}, a$ in $E$, denote by $\nu(d y \times d t)$ the Lévy measure of $\left(\left(\psi_{x_{1}}, \psi_{x_{2}}, \ldots, \psi_{x_{n}}\right), \psi_{a}\right)$. Then one can write:

$$
\nu(d y \times d t)=1_{\{t=0\}} \nu(d y \times\{0\})+1_{\{t>0\}} \nu(d y \times d t) .
$$

Now we look for the infinitely divisible vector corresponding to the Lévy measure $1_{\{t=0\}} \nu(d y \times\{0\})$. We have for every $\alpha_{i}, 1 \leq i \leq n+1$ and $\sigma$ in $\mathbb{R}_{+}$

$$
\begin{aligned}
\mathbb{E}[\exp \{ & \left.\left.-\sum_{i=1}^{n} \alpha_{i} \psi\left(x_{i}\right)-\alpha_{n+1} \psi_{a}-\sigma \psi_{a}\right\}\right] \\
& =\exp \left\{-\int_{\mathbb{R}_{+}^{n}} \int_{0}^{\infty}\left(1-\exp \left\{-\left(\alpha_{n+1}+\sigma\right) t-\sum_{i=1}^{n} \alpha_{i} y_{i}\right\}\right) \nu(d y d t)\right\}
\end{aligned}
$$

In particular, one has:

$$
\mathbb{E}\left[\exp \left\{-\sigma \psi_{a}\right\}\right]=\exp \left\{-\int_{\mathbb{R}_{+}^{n}} \int_{0}^{\infty}(1-\exp \{-\sigma t) \nu(d y d t)\} .\right.
$$

Consequently:

$$
\begin{aligned}
& \frac{\mathbb{E}\left[\exp \left\{-\sum_{i=1}^{n} \alpha_{i} \psi\left(x_{i}\right)-\alpha_{n+1} \psi_{a}-\sigma \psi_{a}\right\}\right]}{\mathbb{E}\left[\exp \left\{-\sigma \psi_{a}\right\}\right]} \\
= & \exp \left\{-\int_{\mathbb{R}_{+}^{n}} \int_{0}^{\infty} e^{-\sigma t}\left(1-\exp \left\{-\alpha_{n+1} t-\sum_{i=1}^{n} \alpha_{i} y_{i}\right\}\right) \nu(d y d t)\right\}
\end{aligned}
$$


which by dominated convergence, as $\sigma$ tends to $+\infty$, converges to

$$
\exp \left\{-\int_{\mathbb{R}_{+}^{n}}\left(1-\exp \left\{-\sum_{i=1}^{n} \alpha_{i} y_{i}\right\}\right) \nu(d y \times\{0\})\right\}
$$

On the other hand, using the fact that if $L(\sigma)$ is the Laplace transform of a nonnegative random variable $Z$ then $\lim _{\sigma \rightarrow \infty} L(\sigma)=\mathbb{P}(Z=0)$, one has:

$$
\frac{\mathbb{E}\left[\exp \left\{-\sum_{i=1}^{n} \alpha_{i} \psi\left(x_{i}\right)-\alpha_{n+1} \psi_{a}-\sigma \psi_{a}\right\}\right]}{\mathbb{E}\left[\exp \left\{-\sigma \psi_{a}\right\}\right]} \longrightarrow_{\sigma \rightarrow \infty} \mathbb{E}\left[\exp \left\{-\sum_{i=1}^{n} \alpha_{i} \psi\left(x_{i}\right)\right\} \mid \psi_{a}=0\right]
$$

Denote by $\phi$ the process $\psi$ conditioned by $\psi(a)=0$. Denote by $\mathcal{L}^{(a)}$ an infinitely divisible nonnegative process with Lévy measure $1_{\{t>0\}} \nu(d y \times d t)$, independent of $\phi$. We hence obtain

$$
\psi \stackrel{(\text { law })}{=} \phi+\mathcal{L}^{(a)} \text {. }
$$

Note that

$$
\left(\mathcal{L}^{(a)} \mid \mathcal{L}^{(a)}(a)=0\right)=0
$$

Proof of Theorem 1.2. We start from (1.1). We know that for every $a$ such that $\mathbb{E}[\psi(a)]>$ 0 , there exists a nonnegative process $r^{(a)}$ independent of $\psi$ such that: $\psi+r^{(a)}$ has the law of $\psi$ under $\mathbb{E}\left[\frac{\psi(a)}{\mathbb{E}[\psi(a)]},.\right]$.

This is a consequence of Lemma 3.1 in [5], but one can check it easily. Indeed, denote by $\mu_{n}$ the Lévy measure of $\left(\psi_{x_{1}}, \psi_{x_{2}}, \ldots, \psi_{x_{n}}\right)$ and assume that $x_{1}=a$, then we have:

$$
\begin{aligned}
& \left.\mathbb{E}\left[\quad \psi(a) \quad \exp \left\{-\sum_{i=1}^{n} \alpha_{i} \psi\left(x_{i}\right)\right\}\right]=-\frac{\partial}{\partial \alpha_{1}} \exp \left\{-\sum_{i=1}^{n} \alpha_{i} \psi\left(x_{i}\right)\right\}\right] \\
& =\mathbb{E}\left[\exp \left\{-\sum_{i=1}^{n} \alpha_{i} \psi\left(x_{i}\right)\right\}\right] \frac{\partial}{\partial \alpha_{1}} \int_{\mathbb{R}_{+}^{n}}\left(1-e^{-\sum_{i=1}^{n} \alpha_{i} y_{i}}\right) \mu_{n}\left(d y_{1} d y_{2} \ldots d y_{n}\right) \\
& =\mathbb{E}\left[\exp \left\{-\sum_{i=1}^{n} \alpha_{i} \psi\left(x_{i}\right)\right\}\right] \int_{\mathbb{R}_{+}^{n}} y_{1} e^{-\sum_{i=1}^{n} \alpha_{i} y_{i}} \mu_{n}\left(d y_{1} d y_{2} \ldots d y_{n}\right) .
\end{aligned}
$$

Hence

$$
\mathbb{E}\left[\exp \left\{-\sum_{i=1}^{n} \alpha_{i} r_{x_{i}}^{(a)}\right\}\right] \mathbb{E}\left[\exp \left\{-\sum_{i=1}^{n} \alpha_{i} \psi\left(x_{i}\right)\right\}\right]=\mathbb{E}\left[\frac{\psi(a)}{\mathbb{E}[\psi(a)]} \exp \left\{-\sum_{i=1}^{n} \alpha_{i} \psi\left(x_{i}\right)\right\}\right],
$$

with

$$
\mathbb{E}\left[\exp \left\{-\sum_{i=1}^{n} \alpha_{i} r_{x_{i}}^{(a)}\right\}\right]=\frac{1}{\mathbb{E}[\psi(a)]} \int_{\mathbb{R}_{+}^{n}} y_{1} e^{-\sum_{i=1}^{n} \alpha_{i} y_{i}} \mu_{n}\left(d y_{1} d y_{2} \ldots d y_{n}\right) .
$$

One also notes that:

$$
\mathbb{P}\left(r_{a}^{(a)} \in d y_{1}\right)=\frac{y_{1}}{\mathbb{E}[\psi(a)]} \int_{\mathbb{R}_{+}^{n-1}} \mu_{n}\left(d y_{1} d y_{2} \ldots d y_{n}\right)
$$

and consequently one obtains

$$
\mathbb{P}\left(r_{a}^{(a)}=0\right)=0
$$

We also have:

$$
\mu_{n}\left(d y_{1} \ldots d y_{n}\right) 1_{\left\{y_{1}>0\right\}}=\mathbb{E}[\psi(a)] \mathbb{E}\left[\frac{1}{r_{a}^{(a)}} ; r_{x_{i}}^{(a)} \in d y_{i}, 1 \leq i \leq n\right]
$$


More generally one obtains

$$
\mu(d y) 1_{\left\{y_{a}>0\right\}}=\mathbb{E}[\psi(a)] \mathbb{E}\left[\frac{1}{r_{a}^{(a)}} ; r^{(a)} \in d y\right] .
$$

Now we rewrite (6.3) under the form:

$$
\frac{\partial}{\partial \alpha_{1}} \log \mathbb{E}\left[\exp \left\{-\sum_{i=1}^{n} \alpha_{i} \psi\left(x_{i}\right)\right\}\right]=-\mathbb{E}[\psi(a)] \mathbb{E}\left[\exp \left\{-\sum_{i=1}^{n} \alpha_{i} r_{x_{i}}^{(a)}\right\}\right] .
$$

Therefore

$$
\frac{\partial}{\partial \alpha_{1}} \log \frac{\mathbb{E}\left[\exp \left\{-\sum_{i=1}^{n} \alpha_{i} \psi\left(x_{i}\right)\right\}\right]}{\mathbb{E}\left[\exp \left\{-\alpha_{1} \psi\left(x_{1}\right)\right\}\right.}=-\mathbb{E}[\psi(a)] \mathbb{E}\left[\left(\exp \left\{-\sum_{i=2}^{n} \alpha_{i} r_{x_{i}}^{(a)}\right\}-1\right) \exp \left\{-\alpha_{1} r_{a}^{(a)}\right\}\right]
$$

Integration with respect to $\alpha_{1}$ on $[0, \sigma]$, gives

$$
\begin{aligned}
\log & \frac{\mathbb{E}\left[\exp \left\{-\sum_{i=2}^{n} \alpha_{i} \psi\left(x_{i}\right)-\sigma \psi(a)\right\}\right]}{\mathbb{E}[\exp \{-\sigma \psi(a)\}]}-\log \mathbb{E}\left[\exp \left\{-\sum_{i=2}^{n} \alpha_{i} \psi\left(x_{i}\right)\right\}\right] \\
& =-\mathbb{E}[\psi(a)] \mathbb{E}\left[\left(\exp \left\{-\sum_{i=2}^{n} \alpha_{i} r_{x_{i}}^{(a)}\right\}-1\right) \frac{\left(1-\exp \left\{-\sigma r_{a}^{(a)}\right\}\right.}{r_{a}^{(a)}} 1_{r_{a}^{(a)}>0}\right] \\
& -\sigma \mathbb{E}\left[\left(\exp \left\{-\sum_{i=2}^{n} \alpha_{i} r_{x_{i}}^{(a)}\right\}-1\right) 1_{r_{a}^{(a)}=0}\right]
\end{aligned}
$$

Since $r_{a}^{(a)}>0$ a.s., the above equation can be rewritten as

$$
\begin{aligned}
\mathbb{E}\left[\exp \left\{-\sum_{i=2}^{n} \alpha_{i} \psi\left(x_{i}\right)\right\}\right] & =\frac{\mathbb{E}\left[\exp \left\{-\sum_{i=2}^{n} \alpha_{i} \psi\left(x_{i}\right)-\sigma \psi(a)\right\}\right]}{\mathbb{E}[\exp \{-\sigma \psi(a)\}]} \\
\times & \exp \left\{-\mathbb{E}[\psi(a)] \mathbb{E}\left[\left(1-\exp \left\{-\sum_{i=2}^{n} \alpha_{i} r_{x_{i}}^{(a)}\right\}\right) \frac{\left(1-\exp \left\{-\sigma r_{a}^{(a)}\right\}\right)}{r_{a}^{(a)}}\right]\right\}
\end{aligned}
$$

The term $\frac{\mathbb{E}\left[\exp \left\{-\sum_{i=2}^{n} \alpha_{i} \psi\left(x_{i}\right)-\sigma \psi(a)\right\}\right]}{\mathbb{E}[\exp \{-\sigma \psi(a)\}]}$ is the Laplace transform of an infinitely divisible nonnegative vector $\left(\psi_{(\sigma)}\left(x_{i}\right), 2 \leq i \leq n\right)$.

Letting $\sigma$ tend to $\infty$, we know that $\psi_{(\sigma)}$ converges in law to $(\psi \mid \psi(a)=0)$ (see the proof of Theorem 1.1), a nonnegative infinitely divisible process with Lévy measure $\nu(d y \times\{0\})$.

Using (6.7), one hence obtains that

$$
\lim _{\sigma \rightarrow \infty} \exp \left\{-\mathbb{E}[\psi(a)] \mathbb{E}\left[\left(1-\exp \left\{-\sum_{i=2}^{n} \alpha_{i} r_{x_{i}}^{(a)}\right\}\right) \frac{\left(1-\exp \left\{-\sigma r_{a}^{(a)}\right\}\right)}{r_{a}^{(a)}}\right]\right\}
$$

exists and is the Laplace tranform of $\left(\mathcal{L}^{(a)}\left(x_{i}\right), 2 \leq i \leq n\right)$. Consequently, the Lévy measure of $\mathcal{L}^{(a)}$ is the law of $r^{(a)}$ under $\mathbb{E}[\psi(a)] \mathbb{E}\left[\frac{1}{r_{a}^{(a)}} ; \quad.\right]$.

The expression (1.2) of the Lévy measure of $\psi$ when $E$ is a separable metric space and $\psi$ is stochastically continuous, is a consequence of Theorem 1.3 (1.3) in the case when $V=E$.

Proof of Theorem 1.3. Again, we start from (1.1). For any $a$ in $E$, any non necessarily distinct $x_{1}, x_{2}, \ldots, x_{n}$ in $E$, and any $\alpha_{1}, \alpha_{2}, . ., \alpha_{n} \geq 0$

$$
\mathbb{E}[\psi(a)] \mathbb{E}\left[\exp \left\{-\sum_{i=1}^{n} \alpha_{i} r_{x_{i}}^{(a)}\right\}\right] \mathbb{E}\left[\exp \left\{-\sum_{i=1}^{n} \alpha_{i} \psi\left(x_{i}\right)\right\}\right]=\mathbb{E}\left[\psi(a) \exp \left\{-\sum_{i=1}^{n} \alpha_{i} \psi\left(x_{i}\right)\right\}\right] .
$$


For any real function $f$ on $E$, set: $I(f)=\int_{V} f(x) m(d x)$. We have:

$$
\begin{array}{r}
\mathbb{E}[\psi(a)] \mathbb{E}\left[\exp \left\{-\alpha_{1} I\left(r^{(a)}\right)-\sum_{i=2}^{n} \alpha_{i} r_{x_{i}}^{(a)}\right\}\right] \mathbb{E}\left[\exp \left\{-\alpha_{1} I(\psi)-\sum_{i=2}^{n} \alpha_{i} \psi\left(x_{i}\right)\right\}\right] \\
=\mathbb{E}\left[\psi(a) \exp \left\{-\alpha_{1} I(\psi)-\sum_{i=2}^{n} \alpha_{i} \psi\left(x_{i}\right)\right\}\right],
\end{array}
$$

which leads, after integration of each member over $V$ with respect to $m(d a)$, to

$$
\begin{aligned}
-\int_{E} \mathbb{E}[\psi(a)]\left[\operatorname { e x p } \left\{-\alpha_{1} I\left(r^{(a)}\right)\right.\right. & \left.\left.-\sum_{i=2}^{n} \alpha_{i} r_{x_{i}}^{(a)}\right\}\right] m(d a) \\
& =\frac{\partial}{\partial \alpha_{1}} \log \mathbb{E}\left[\exp \left\{-\alpha_{1} I(\psi)-\sum_{i=2}^{n} \alpha_{i} \psi\left(x_{i}\right)\right\}\right] .
\end{aligned}
$$

One obtains:

$$
\begin{aligned}
\frac{\partial}{\partial \alpha_{1}} \log \mathbb{E}\left[\operatorname { e x p } \left\{-\alpha_{1} I(\psi)\right.\right. & \left.\left.-\sum_{i=2}^{n} \alpha_{i} \psi\left(x_{i}\right)\right\}\right] \\
& =-\int_{V} \mathbb{E}[\psi(a)] \mathbb{E}\left[\exp \left\{-\alpha_{1} I\left(r^{(a)}\right)-\sum_{i=2}^{n} \alpha_{i} r_{x_{i}}^{(a)}\right\}\right] m(d a)
\end{aligned}
$$

Therefore

$$
\begin{aligned}
\frac{\partial}{\partial \alpha_{1}} \quad \log \frac{\mathbb{E}\left[\exp \left\{-\alpha_{1} I(\psi)-\sum_{i=2}^{n} \alpha_{i} \psi\left(x_{i}\right)\right\}\right]}{\mathbb{E}\left[\exp \left\{-\alpha_{1} I(\psi)\right\}\right]} \\
=-\int_{V} \mathbb{E}[\psi(a)] \mathbb{E}\left[\left(\exp \left\{-\sum_{i=2}^{n} \alpha_{i} r_{x_{i}}^{(a)}\right\}-1\right) \exp \left\{-\alpha_{1} I\left(r^{(a)}\right)\right\}\right] m(d a) .
\end{aligned}
$$

Integration with respect to $\alpha_{1}$ on $[0, \sigma]$, gives

$$
\begin{aligned}
& \log \frac{\mathbb{E}\left[\exp \left\{-\sum_{i=2}^{n} \alpha_{i} \psi\left(x_{i}\right)-\sigma I(\psi)\right\}\right]}{\mathbb{E}[\exp \{-\sigma I(\psi)\}]}-\log \mathbb{E}\left[\exp \left\{-\sum_{i=2}^{n} \alpha_{i} \psi\left(x_{i}\right)\right\}\right] \\
& =-\int_{V} \mathbb{E}[\psi(a)] \mathbb{E}\left[\left(\exp \left\{-\sum_{i=2}^{n} \alpha_{i} r_{x_{i}}^{(a)}\right\}-1\right) \frac{\left(1-\exp \left\{-\sigma I\left(r^{(a)}\right)\right\}\right.}{I\left(r^{(a)}\right)} 1_{I\left(r^{(a)}\right)>0}\right] m(d a) \\
& -\quad \sigma \int_{V} \mathbb{E}[\psi(a)] \mathbb{E}\left[\left(\exp \left\{-\sum_{i=2}^{n} \alpha_{i} r_{x_{i}}^{(a)}\right\}-1\right) 1_{I\left(r^{(a)}\right)=0}\right] m(d a)
\end{aligned}
$$

and equivalently:

$$
\begin{aligned}
& \mathbb{E}\left[\exp \left\{-\sum_{i=2}^{n} \alpha_{i} \psi\left(x_{i}\right)\right\}\right]=\frac{\mathbb{E}\left[\exp \left\{-\sum_{i=2}^{n} \alpha_{i} \psi\left(x_{i}\right)-\sigma I(\psi)\right\}\right]}{\mathbb{E}[\exp \{-\sigma I(\psi)\}]} \\
\times & \exp \left\{-\int_{V} \mathbb{E}[\psi(a)] \mathbb{E}\left[\left(1-\exp \left\{-\sum_{i=2}^{n} \alpha_{i} r_{x_{i}}^{(a)}\right\}\right) \frac{\left(1-\exp \left\{-\sigma I\left(r^{(a)}\right)\right\}\right.}{I\left(r^{(a)}\right)} 1_{I\left(r^{(a)}\right)>0}\right] m(d a)\right\} \\
\times & \exp \left\{-\sigma \int_{V} \mathbb{E}[\psi(a)] \mathbb{E}\left[\left(1-\exp \left\{-\sum_{i=2}^{n} \alpha_{i} r_{x_{i}}^{(a)}\right\}\right) 1_{I\left(r^{(a)}\right)=0}\right] m(d a)\right\} .
\end{aligned}
$$

Denote by $\phi_{\sigma}$ a nonnegative infinitely divisible process satisfying

$$
\mathbb{E}\left[\exp \left\{-\sum_{i=2}^{n} \alpha_{i} \phi_{\sigma}\left(x_{i}\right)\right\}\right]=\frac{\mathbb{E}\left[\exp \left\{-\sum_{i=2}^{n} \alpha_{i} \psi\left(x_{i}\right)-\sigma I(\psi)\right\}\right]}{\mathbb{E}[\exp \{-\sigma I(\psi)\}]} .
$$


On one hand, with elementary properties of the Laplace transform of nonnegative random variables, we have:

$$
\frac{\mathbb{E}\left[\exp \left\{-\sum_{i=2}^{n} \alpha_{i} \psi\left(x_{i}\right)-\sigma I(\psi)\right\}\right]}{\mathbb{E}[\exp \{-\sigma I(\psi)\}]} \rightarrow_{\sigma \rightarrow \infty} \mathbb{E}\left[\exp \left\{-\sum_{i=2}^{n} \alpha_{i} \psi\left(x_{i}\right)\right\} \mid I(\psi)=0\right] .
$$

On the other hand note that $(\psi, I(\psi))$ is infinitely divisible. Denote by $\nu(d y d t)$ the Lévy measure of $\left(\left(\psi\left(x_{i}\right)_{2 \leq i \leq n}, I(\psi)\right)\right.$, then we have:

$$
\begin{aligned}
\mathbb{E}[\quad \exp & \left.-\left\{\sum_{i=2}^{n} \alpha_{i} \psi\left(x_{i}\right)+\alpha_{1} I(\psi)+\sigma I(\psi)\right\}\right] \\
& =\exp \left\{-\int_{\mathbb{R}_{+}^{n-1}} \int_{0}^{\infty}\left(1-\exp \left\{-\left(\alpha_{1}+\sigma\right) t-\sum_{i=2}^{n} \alpha_{i} y_{i}\right\}\right) \nu(d y d t)\right\}
\end{aligned}
$$

In particular, one has:

$$
\mathbb{E}[\exp \{-\sigma I(\psi)\}]=\exp \left\{-\int_{\mathbb{R}_{+}^{n-1}} \int_{0}^{\infty}(1-\exp \{-\sigma t) \nu(d y d t)\} .\right.
$$

Consequently:

$$
\begin{aligned}
& \frac{\mathbb{E}\left[\exp \left\{-\sum_{i=2}^{n} \alpha_{i} \psi\left(x_{i}\right)-\alpha_{1} I(\psi)-\sigma I(\psi)\right\}\right]}{\mathbb{E}[\exp \{-\sigma I(\psi)\}]} \\
= & \exp \left\{-\int_{\mathbb{R}_{+}^{n-1}} \int_{0}^{\infty} e^{-\sigma t}\left(1-\exp \left\{-\alpha_{1} t-\sum_{i=2}^{n} \alpha_{i} y_{i}\right\}\right) \nu(d y d t)\right\},
\end{aligned}
$$

which by dominated convergence, converges, as $\sigma$ tends to $+\infty$, to

$$
\exp \left\{-\int_{\mathbb{R}_{+}^{n-1}}\left(1-\exp \left\{-\sum_{i=2}^{n} \alpha_{i} y_{i}\right\}\right) \nu(d y \times\{0\})\right\} .
$$

Consequently $\phi_{\sigma}$ converges to an infinitely divisible nonnegative process $\phi_{\infty}$ with Lévy measure $\nu(d y \times\{0\})$ and by (6.9): $\phi_{\infty} \stackrel{\text { (law) }}{=}(\psi \mid I(\psi)=0)$.

In view of (6.8), this implies that

$$
\begin{aligned}
\lim _{\sigma \rightarrow \infty}\left(\exp \left\{-\int_{V} \mathbb{E}[\psi(a)] \mathbb{E}\left[\left(1-\exp \left\{-\sum_{i=2}^{n} \alpha_{i} r_{x_{i}}^{(a)}\right\}\right) \frac{\left(1-\exp \left\{-\sigma I\left(r^{(a)}\right)\right\}\right.}{I\left(r^{(a)}\right)} 1_{I\left(r^{(a)}\right)>0}\right] m(d a)\right\}\right. \\
\left.\quad \times \exp \left\{-\sigma \int_{V} \mathbb{E}[\psi(a)] \mathbb{E}\left[\left(1-\exp \left\{-\sum_{i=2}^{n} \alpha_{i} r_{x_{i}}^{(a)}\right\}\right) 1_{I\left(r^{(a)}\right)=0}\right) m(d a)\right\}\right)
\end{aligned}
$$

is the Laplace transform of a nonnegative infinitely divisible vector with Lévy measure $\int_{\mathbb{R}+} 1_{t>0} \nu(d y d t)$. Consequently, we must have:

$$
\int_{V} \mathbb{E}[\psi(a)] \mathbb{E}\left[\left(1-\exp \left\{-\sum_{i=2}^{n} \alpha_{i} r_{x_{i}}^{(a)}\right\}\right) 1_{I\left(r^{(a)}\right)=0}\right] m(d a)=0
$$

and

$$
\lim _{\sigma \rightarrow \infty} \exp \left\{-\int_{V} \mathbb{E}[\psi(a)] \mathbb{E}\left[\left(1-\exp \left\{-\sum_{i=2}^{n} \alpha_{i} r_{x_{i}}^{(a)}\right\}\right) \frac{\left(1-\exp \left\{-\sigma I\left(r^{(a)}\right)\right\}\right.}{I\left(r^{(a)}\right)}\right] m(d a)\right\}
$$

is the Laplace transform of a nonnegative infinitely divisible vector with Lévy measure $\int_{\mathbb{R}+} 1_{t>0} \nu(d y d t)$. 
By monotone convergence, one obtains:

$$
\int_{\mathbb{R}+} 1_{t>0} \nu(d y d t)=\int_{V} \mathbb{E}[\psi(a)] \mathbb{E}\left[\frac{1}{I\left(r^{(a)}\right)},\left(r_{x_{i}}^{(a)}\right)_{2 \leq i \leq n} \in d y\right] m(d a) .
$$

Moreover there exists an infinitely divisible nonnegative process $\mathcal{L}^{V}$ with Lévy measure $\int_{V} \mathbb{E}[\psi(a)] \mathbb{E}\left[\frac{1}{I\left(r^{(a)}\right)},\left(r_{x_{i}}^{(a)}\right)_{2 \leq i \leq n} \in d y\right] m(d a)$, independent of $\phi_{\infty}$ such that

$$
\psi \stackrel{(\text { law })}{=} \phi_{\infty}+\mathcal{L}^{V}
$$

Note that any version of $\phi_{\infty}$ satisfies (6.10).

Assume now that $E$ is a separable metric space and $\psi$ is stochastically continuous. We show that $\phi_{\infty}=0$ on $V$.

We know that $\int_{V} \phi_{\infty}(x) m(d x)=0$ a.s., hence $\mathbb{E}\left[\int_{V} \phi_{\infty}(x) m(d x)\right]=0$, which implies that there exists a subset $S$ of $V$ such that $m(V \backslash S)=0$ and for every $x$ in $S: \mathbb{E}\left[\phi_{\infty}(x)\right]=0$. Consequently there exists a version of $\phi_{\infty}$ which is identically equal to 0 on $S$. We still denote this version by $\phi_{\infty}$.

The subset $S$ is dense in $V$. Indeed, let $a$ be in $V \backslash S$. Since $V$ is open there exists $\varepsilon>0$ such that the open ball $B(a, \varepsilon)$ is included in $V$. Hence for every integer $n, B\left(a, \frac{\varepsilon}{n}\right)$ is included in $V$. Necessarely: $m\left(B\left(a, \frac{\varepsilon}{n}\right)>0\right.$. This implies that: $B\left(a, \frac{\varepsilon}{n}\right) \cap S \neq \emptyset$. We choose $s_{n}$ in $B\left(a, \frac{\varepsilon}{n}\right) \cap S$ and obtain a sequence $\left(s_{n}\right)$ converging to $a$.

Since $\psi$ is stochastically continuous $\left(\psi_{s_{n}}\right)$ tends to $\psi_{a}$ in probability. This implies that $\left(\mathcal{L}_{s_{n}}^{V}-\mathcal{L}_{a}^{V}\right)$ tends to $\phi_{\infty}(a)$ in probability. Since $\phi_{\infty}(a)$ is independent of the sequence $\left(\mathcal{L}_{x_{n}}^{V}-\mathcal{L}_{a}^{V}\right)$, the variable $\phi_{\infty}(a)$ must be deterministic a.s. By (6.10), the random variable $\psi_{a}$ is hence the sum of a nonnegative constant and a nonnegative infinitely divisible random variable. We have assumed that $\psi$ has no drift hence $\phi_{\infty}(a)=0$ a.s. Consequently $\phi_{\left.\infty\right|_{V}}$ admits an identically equal to 0 version. This establishes (1.3).

To establish Theorem 1.4 we first establish the following lemma which gives the expression of $g_{T_{a}}$.

Lemma 6.1. For $x, y$ in $E$, we have:

$$
g_{T_{a}}(x, y)=g(x, y)-\frac{g(x, a) g(a, y)}{g(a, a)} .
$$

Proof of Lemma 6.1. $g(x, y)=\mathbb{E}_{x}\left[L_{\infty}^{y}\right]=\mathbb{E}_{x}\left[L_{\infty}^{y} ; T_{a}=\infty\right]+\mathbb{E}_{x}\left[L_{\infty}^{y} ; T_{a}<\infty\right]$, and

$$
\mathbb{E}_{x}\left[L_{\infty}^{y} ; T_{a}<\infty\right]=\mathbb{E}_{x}\left[L_{T_{a}}^{y}+L_{\infty}^{y} \circ \theta_{T_{a}} ; T_{a}<\infty\right]=\mathbb{E}_{x}\left[L_{T_{a}}^{y} ; T_{a}<\infty\right]+\mathbb{P}\left[T_{a}<\infty\right] \mathbb{E}_{x}\left[L_{\infty}^{y}\right] .
$$

Hence: $g(x, y)=g_{T_{a}}(x, y)+\mathbb{P}_{x}\left[T_{a}<\infty\right) g(a, y)$. To conclude, one finally notes that: $\mathbb{P}_{x}\left[T_{a}<\infty\right]=\frac{g(x, a)}{g(a, a)}$.

Proof of Theorem 1.4. We have established in [7] that for every $a$ such that $\mathbb{E}[\psi(a)]>0$, we have:

$$
\frac{1}{2} \psi+L^{(a)} \stackrel{\text { (law) }}{=} \frac{1}{2} \psi \text { under } \mathbb{E}\left[\frac{\psi(a)}{\mathbb{E}[\psi(a)]}, .\right]
$$

Hence thanks to Theorems 1.1 and 1.2, we know that there exists an infinitely divisible nonnegative process $\mathcal{L}^{(a)}$, independent of $\psi$, with Lévy measure the law of $L^{(a)}$ under $\frac{\mathbb{E}[\psi(a)]}{2} \mathbb{E}\left[\frac{1}{L_{a}^{(a)}} ; \quad.\right]$ such that:

$$
\frac{1}{2} \psi \stackrel{(\text { law })}{=} \frac{1}{2}(\psi \mid \psi(a)=0)+\mathcal{L}^{(a)} .
$$


Making use of (6.7) in the proof of Theorem 1.1, we also know that for every such fixed $a$, and for every $\sigma>0$ there exists an infinitely divisible nonnegative process $\ell_{(\sigma)}$ with Lévy measure the law of $L^{(a)}$ under $\mathbb{E}\left[\frac{g(a, a)}{2} \frac{\left(1-\exp \left\{-\sigma L_{a}^{(a)}\right\}\right.}{L_{a}^{(a)}} ;\right.$. ] such that

$$
\frac{1}{2} \psi \stackrel{(\text { law })}{=} \frac{1}{2} \phi_{(\sigma)}+\ell_{(\sigma)}
$$

where $\phi_{(\sigma)}$ is independent of $\ell_{(\sigma)}$, and has the law of $\psi$ under $\mathbb{E}\left[\frac{\exp \left\{-\frac{\sigma}{2} \psi(a)\right\}}{\mathbb{E}\left[\exp \left\{-\frac{1}{2} \sigma \psi(a)\right\}\right.} ;.\right]$.

Moreover we know that

$$
\mathbb{E}\left[\exp \left\{-\frac{1}{2} \sum_{i=2}^{n} \alpha_{i} \psi\left(x_{i}\right)\right\} \mid \psi(a)=0\right]=\lim _{\sigma \rightarrow \infty} \frac{\mathbb{E}\left[\exp \left\{-\frac{1}{2} \sum_{i=2}^{n} \alpha_{i} \psi\left(x_{i}\right)-\frac{\sigma}{2} \psi(a)\right\}\right]}{\mathbb{E}\left[\exp \left\{-\frac{1}{2} \sigma \psi(a)\right\}\right]}
$$

We will now identify $(\psi \mid \psi(a)=0)$ and $\mathcal{L}^{(a)}$.

On one hand:

$$
\frac{\mathbb{E}\left[\exp \left\{-\frac{1}{2} \sum_{i=2}^{n} \alpha_{i} \psi\left(x_{i}\right)-\frac{\sigma}{2} \psi(a)\right\}\right]}{\mathbb{E}\left[\exp \left\{-\frac{1}{2} \sigma \psi(a)\right\}\right]}
$$

is the Laplace transform of a permanental vector (see for example [12]) with index 2 and kernel $g^{(\sigma)}$ defined by:

$$
g^{(\sigma)}(x, y)=g(x, y)-\frac{\sigma}{1+\sigma g(a, a)} g(x, a) g(a, y)
$$

By letting $\sigma$ tend to $\infty$ in (6.14), using Lemma 6.1, one sees that $\psi_{(\sigma)}$ converges in law to the permanental vector with kernel $g_{T_{a}}$ and index 2 and consequently

$$
(\psi \mid \psi(a)=0) \stackrel{(\text { law })}{=} \psi_{g_{T_{a}}}
$$

On the other hand, from its definition, the Laplace transform of $\psi_{(\sigma)}$ satisfies

$$
\mathbb{E}\left[\exp \left\{-\frac{1}{2} \sum_{i=2}^{n} \alpha_{i} \psi_{(\sigma)}\left(x_{i}\right)\right\}\right]=\left(\frac{1+\sigma g(a, a)}{\operatorname{det}\left(I+D_{\sigma} G\right)}\right)^{1 / 2}
$$

where $G=\left(g\left(x_{i}, x_{j}\right)\right)_{1 \leq i, j \leq n}$ and $D_{\sigma}$ is the diagonal matrix with diagonal entries $\left(\sigma, \alpha_{2}, \ldots, \alpha_{n}\right)$. Developing $\operatorname{det}\left(I+D_{\sigma} G\right)$ with respect to its first row gives

$$
\begin{aligned}
\operatorname{det}(I+ & \left.D_{\sigma} G\right) \\
= & (1+\sigma g(a, a))\left(I+D_{\sigma} G\right)^{11}-\sigma g\left(a, x_{2}\right)\left(I+D_{\sigma} G\right)^{12} \\
& +\sigma g\left(a, x_{3}\right)\left(I+D_{\sigma} G\right)^{13}+\ldots+(-1)^{n+1} \sigma g\left(a, x_{n}\right)\left(I+D_{\sigma} G\right)^{1 n} \\
= & (1+\sigma g(a, a))\left(I+D_{0} G\right)^{11}-\sigma g\left(a, x_{2}\right)\left(I+D_{0} G\right)^{12} \\
& +\sigma g\left(a, x_{3}\right)\left(I+D_{0}\right)^{13}+\ldots+(-1)^{n+1} \sigma g\left(a, x_{n}\right)\left(I+D_{0}\right)^{1 n}
\end{aligned}
$$

hence the limit in (6.16) when $\sigma$ tends to $\infty$ is equal to

$$
\left(\frac{g(a, a)}{\operatorname{det} V}\right)^{1 / 2}
$$

where $V=\left(V_{i, j}\right)_{1 \leq i, j \leq n}$ with $V_{i j}=\left(I+D_{0}\right)_{i j}$ when $i \neq 1$ and $V_{1 j}=g\left(a, x_{j}\right)$.

Consequently, one obtains by letting $\sigma$ tend to $\infty$ in (6.13)

$$
\operatorname{det}\left(I+D_{0} G\right)^{-1 / 2}\left(\frac{g(a, a)}{\operatorname{det} V}\right)^{-1 / 2}=\lim _{\sigma \rightarrow \infty} \mathbb{E}\left[\exp \left\{-\sum_{i=2}^{n} \alpha_{i} \ell_{(\sigma)}\left(x_{i}\right)\right\}\right]
$$


But note that

$$
\begin{aligned}
\frac{\partial}{\partial \sigma}\left(\operatorname{det}\left(I+D_{\sigma} G\right)\right)^{-1 / 2} & =-\frac{1}{2}\left(\operatorname{det}\left(I+D_{\sigma} G\right)\right)^{-3 / 2} \frac{\partial}{\partial \sigma}\left(\operatorname{det}\left(I+D_{\sigma} G\right)\right) \\
& =-\frac{1}{2}\left(\operatorname{det}\left(I+D_{\sigma} G\right)\right)^{-3 / 2} \operatorname{det} V
\end{aligned}
$$

and also that

$$
\begin{aligned}
\frac{\partial}{\partial \sigma}\left(\operatorname{det}\left(I+D_{\sigma} G\right)\right)^{-1 / 2} & =\frac{\partial}{\partial \sigma} \mathbb{E}\left[\exp \left\{-\frac{1}{2} \sum_{i=2}^{n} \alpha_{i} \psi\left(x_{i}\right)-\frac{\sigma}{2} \psi(a)\right\}\right] \\
& =-\frac{1}{2} \mathbb{E}\left[\psi(a) \exp \left\{-\frac{1}{2} \sum_{i=2}^{n} \alpha_{i} \psi\left(x_{i}\right)-\frac{1}{2} \sigma \psi\left(x_{1}\right)\right\}\right]
\end{aligned}
$$

which together lead to:

$$
\mathbb{E}\left[\psi(a) \exp \left\{-\frac{1}{2} \sum_{i=2}^{n} \alpha_{i} \psi\left(x_{i}\right)-\frac{1}{2} \sigma \psi\left(x_{1}\right)\right\}\right]=\left(\operatorname{det}\left(I+D_{\sigma} G\right)\right)^{-3 / 2} \operatorname{det} V .
$$

Making use of both (6.11) and (6.18), (6.17) translates into:

$$
\lim _{\sigma \rightarrow \infty} \mathbb{E}\left[\exp \left\{-\sum_{i=2}^{n} \alpha_{i} \ell_{(\sigma)}\left(x_{i}\right)\right\}\right]=\tilde{\mathbb{E}}_{a}\left[\exp \left\{-\sum_{i=2}^{n} \alpha_{i} L_{\infty}^{x_{i}}\right\}\right]^{1 / 2},
$$

which leads to Theorem 1.4.

Proof of Theorem 1.5. This theorem is a direct consequence of Theorem 1.2 and (6.11). The only thing that we need to show is (1.6) without the assumption of stochastic continuity required by (1.2).

Define $\mu_{1}$ as the Lévy measure of $\left(\left(\frac{1}{2} \psi_{x_{i}}, 1 \leq i \leq n\right), \frac{1}{2} \int_{E} \psi(x) m(d x)\right)$. In the proof of Corollary 3.3 (p.1411, 1.4 [7]), we have established:

$$
y_{a} \mu_{(n)}(d y)=\frac{g(a, a)}{2} \tilde{\mathbb{P}}_{a}\left[L_{\infty}^{a} \in d y_{a}, L_{\infty}^{x_{i}} \in d y_{x_{i}}, 2 \leq i \leq n\right],
$$

where $\mu_{(n)}$ is the Lévy measure of the vector $\left(\frac{1}{2} \psi_{x_{i}}, 1 \leq i \leq n\right)$.

Using the fact that: $\int_{E} L_{\infty}^{x} m(d x)=\zeta$, one obtains similarly to (6.19):

$$
y_{a} \mu_{1}(d y d t)=\frac{g(a, a)}{2} \tilde{\mathbb{P}}_{a}\left[L_{\infty}^{a} \in d y_{a}, L_{\infty}^{x_{i}} \in d y_{x_{i}}, 2 \leq i \leq n, \zeta \in d t\right]
$$

which leads immediately to:

$$
y_{a} \mu_{1}(d y \times\{0\})=0 .
$$

We show now that: $\mu_{1}(d y \times\{0\})=0$. Suppose that $\mu_{1}(d y \times\{0\})>0$. Then using (6.20), one has: $\mu_{1}(d y \times\{0\})=1_{y_{a}=0} \mu_{1}(d y \times\{0\})$, for every $a$ in $\left\{x_{i}, 1 \leq i \leq n\right\}$, and hence: $\mu_{1}(d y \times\{0\})=1_{y_{x_{i}}=0,1 \leq i \leq n} \mu_{1}(d y \times\{0\})=\mu_{1}\left(\left\{0_{\mathbb{R}^{n+1}}\right\}\right)=0$.

Integrating both sides of (6.11) with respect to $m$ one obtains:

$$
\begin{aligned}
\mathbb{E} & {\left[\int_{E} \psi(a) \exp \left\{-\frac{1}{2} \sum_{i=1}^{n} \alpha_{i} \psi\left(x_{i}\right)\right\} m(d a)\right] } \\
= & \int_{E} g(a, a) \tilde{\mathbb{E}}_{a}\left[\exp \left\{-\sum_{i=1}^{n} \alpha_{i} L_{\infty}^{x_{i}}\right\}\right] \mathbb{E}\left[\exp \left\{-\frac{1}{2} \sum_{i=1}^{n} \alpha_{i} \psi\left(x_{i}\right)\right\}\right] m(d a),
\end{aligned}
$$

from which it follows in the same manner as (6.19) has been obtained that:

$$
t \mu_{1}(d y d t)=\frac{1}{2} \int_{E} g(a, a) \tilde{\mathbb{P}}_{a}\left[L_{\infty}^{a} \in d y_{a}, L_{\infty}^{x_{i}} \in d y_{x_{i}}, 2 \leq i \leq n, \zeta \in d t\right] m(d a) .
$$


Since: $\mu_{1}(d y \times\{0\})=0$, one equivalently has:

$$
\mu_{1}(d y d t)=\int_{E} \frac{g(a, a)}{2} \tilde{\mathbb{P}}_{a}\left[\frac{1}{\zeta}, L_{\infty}^{a} \in d y_{a}, L_{\infty}^{x_{i}} \in d y_{x_{i}}, 2 \leq i \leq n, \zeta \in d t\right] m(d a) .
$$

Integrating with respect to $t$ each member of the above equation we finally obtain

$$
\mu_{(n)}(F)=\int_{E} \frac{g(a, a)}{2} \tilde{\mathbb{P}}_{a}\left[\frac{F\left(L_{\infty}\right)}{\zeta}\right] m(d a),
$$

for every measurable function $F$ on $\mathbb{R}_{+}^{n}$, which leads to (1.6).

Proof of Theorem 1.8. By Theorem 1.3 we know that

$$
\frac{1}{2} \psi \stackrel{(\text { law })}{=} \frac{1}{2}\left(\psi \mid \int_{V} \psi(x) \nu_{A}(d x)=0\right)+\mathcal{L}^{V}
$$

where $\mathcal{L}^{V}$ is an infinitely divisible nonnegative process, independent of $\psi$, with Lévy measure the law of $L_{\infty}$ under $\int_{V} \mathbb{E}\left[\frac{\psi(a)}{2}\right] \tilde{\mathbb{E}}_{a}\left[\frac{1}{\int_{V} L_{\infty}^{x} \nu_{A}(d x)} ;.\right] \nu_{A}(d a)$.

We also know that the Lévy measure of $\frac{1}{2}\left(\psi \mid \int_{V} \psi(x) \nu_{A}(d x)=0\right)$ is $1_{\left\{\int_{V} y(x) \nu(d x)=0\right\}} \mu(d y)$ and that the Lévy measure of $\mathcal{L}^{V}$ is $1_{\left\{\int_{V} y(x) \nu(d x)>0\right\}} \mu(d y)$.

Let $m$ be a measure with support equal to $E$ such that $\int_{E} g(x, x) m(d x)<\infty$, and $m_{\left.\right|_{V}}=\nu_{A}$.

We now interpret $\phi=\left(\psi \mid \int_{V} \psi(x) \nu_{A}(d x)=0\right)$. By Theorem 1.5, we know that $\frac{1}{2} \phi$ is infinitely divisible with Lévy measure the law of $\left(L_{\infty}^{x}, x \in E\right)$ under $\frac{1}{2} \int_{E} g(a, a) \tilde{\mathbb{E}}_{a}\left[\frac{1}{\int_{E} L_{\infty}^{y} m(d y)} 1_{\int_{V} L_{\infty}^{y} \nu_{A}(d y)=0} ; \cdot\right] m(d a)$. Since we have assumed that $V$ is the fine support of $\left(A_{t}\right)_{t \geq 0}$, one obtains that $\left(L_{\infty}^{x}, x \in V\right)$ under $\frac{1}{2} \int_{E} g(a, a) \tilde{\mathbb{E}}_{a}\left[\frac{1}{\int_{E} L_{\infty}^{y} m(d y)} \times\right.$ $1_{\int_{V} L_{\infty}^{y} \nu_{A}(d y)=0} ;$. $] m(d a)$ is identically equal to 0 and that the Lévy measure of $\frac{1}{2} \phi$ is the law of $\left(L_{\infty}^{x}, x \in E\right)$ under $\frac{1}{2} \int_{V^{c}} g(a, a) \tilde{\mathbb{E}}_{a}\left[\frac{1}{\int_{V^{c}} L_{\infty}^{y} m(d y)} 1_{T_{V}=\infty} ;.\right] m(d a)$.

Consequently: $\phi_{\left.\right|_{V}}=0$ and hence $\mathcal{L}_{\left.\right|_{V}} \stackrel{\text { (law) }}{=} \psi_{\left.\right|_{V}}$.

Note that for every bounded $\mathcal{F}_{t}$-measurable variable $F$ and every $a$ in $E$ :

$$
g(a, a) \tilde{\mathbb{E}}_{a}[F]=\mathbb{E}_{a}\left[g\left(X_{t}, a\right), F\right]=\mathbb{E}_{a}\left[L_{\infty}^{a} \circ \theta_{t}, F\right]
$$

In particular, we have, since $\left\{T_{V}>t\right\}$ is in $\mathcal{F}_{t}$ :

$$
g(a, a) \tilde{\mathbb{E}}_{a}\left[F, T_{V}>t\right]=\mathbb{E}_{a}\left[L_{\infty}^{a} \circ \theta_{t}, F, T_{V}>t\right]
$$

Write: $L_{\infty}^{a}=L_{T_{V}}^{a}+\left(L_{\infty}^{a}-L_{T_{V}}^{a}\right)$, then

$$
\begin{aligned}
g(a, a) & \tilde{\mathbb{E}}_{a}\left[F, T_{V}>t\right] \\
= & \mathbb{E}_{a}\left[L_{T_{V}}^{a} \circ \theta_{t}, F, T_{V}>t\right]+\mathbb{E}_{a}\left[\left(L_{\infty}^{a}-L_{T_{V}}^{a}\right) \circ \theta_{t}, F, T_{V}>t\right] \\
= & \mathbb{E}_{a}^{V}\left[L_{\infty}^{a} \circ \theta_{t}, F\right]+\mathbb{E}_{a}\left[\left(L_{\infty}^{a}-L_{T_{V}}^{a}\right) \circ \theta_{t}, F, T_{V}>t\right]
\end{aligned}
$$

where $\mathbb{E}_{a}^{V}$ is the expectation of the law of the Markov process $X$ starting at $a$ and killed at $T_{V}$.

Now using (6.21) for $X$ killed at $T_{V}$, one has:

$$
\mathbb{E}_{a}^{V}\left[L_{\infty}^{a} \circ \theta_{t}, F\right]=g_{T_{V}}(a, a) \tilde{\mathbb{E}}_{a}^{V}[F]
$$

On the other hand, note that:

$$
\mathbb{E}_{a}\left[\left(L_{\infty}^{a}-L_{T_{V}}^{a}\right) \circ \theta_{t}, F, T_{V}>t\right]=\mathbb{E}_{a}\left[\left(L_{\infty}^{a}-L_{T_{V}}^{a}\right) \circ \theta_{t}, F, t<T_{V}<\infty\right]
$$


Together (6.22), (6.23) and (6.24) lead for every $t>0$ to:

$$
\begin{aligned}
g(a, a) \tilde{\mathbb{E}}_{a}\left[F, T_{V}>t\right] & =g_{T_{V}}(a, a) \tilde{\mathbb{E}}_{a}^{V}[F] \\
& +\mathbb{E}_{a}\left[\left(L_{\infty}^{a}-L_{T_{V}}^{a}\right) \circ \theta_{t}, F, t<T_{V}<\infty\right] .
\end{aligned}
$$

In particular for every $\delta>0$ such that $\delta \leq t$ and every bounded $\mathcal{F}_{\delta}$-measurable variable $G$, we have:

$$
\begin{aligned}
g(a, a) \tilde{\mathbb{E}}_{a}\left[G, T_{V}>t\right] & =g_{T_{V}}(a, a) \tilde{\mathbb{E}}_{a}^{V}[G] \\
& +\mathbb{E}_{a}\left[\left(L_{\infty}^{a}-L_{T_{V}}^{a}\right) \circ \theta_{t}, G, t<T_{V}<\infty\right] .
\end{aligned}
$$

On $\left\{T_{V}>t\right\}$, we have: $\left(L_{\infty}^{a}-L_{T_{V}}^{a}\right) \circ \theta_{t}=L_{\infty}^{a} \circ \theta_{T_{V}}$. By conditioning on $\mathcal{F}_{T_{V}}$, one hence has:

$$
\mathbb{E}_{a}\left[\left(L_{\infty}^{a}-L_{T_{V}}^{a}\right) \circ \theta_{t}, G, t<T_{V}<\infty\right]=\mathbb{E}_{a}\left[g\left(X_{T_{V}}, a\right), G, t<T_{V}<\infty\right] .
$$

Since $G$ is bounded and for every $x$ in $E: g(x, a) \leq g(a, a)<\infty$, one obtains by dominated convergence as $t$ tends to $\infty$ :

$$
g(a, a) \tilde{\mathbb{E}}_{a}\left[G, T_{V}=\infty\right]=g_{T_{V}}(a, a) \tilde{\mathbb{E}}_{a}^{V}[G]
$$

which implies by identification from the Lévy measure that: $\phi^{(\text {law })}=\psi_{g_{T_{V}}}$.

\section{References}

[1] Barlow, M. T.: Necessary and sufficient conditions for the continuity of local time of Lévy processes. Ann. Probab. 16, no. 4, 1389-1427 (1988). MR-0958195

[2] Dynkin E. B.: Local times and quantum fields. Seminar on Stochastic Processes, 6-84. Birkhauser (1983). MR-0902412

[3] Eisenbaum N.: Dynkin's isomorphism theorem and the Ray-Knight theorems. Probab. Theory Relat. Fields 99, 321-335 (1994). MR-1278888

[4] Eisenbaum N.: Une version sans conditionnement du Théorème d'isomorphisme de Dynkin. Séminaire de probabilités, XXIX, Lect. Notes in Math., 266-289 Springer (1995). MR-1459468

[5] Eisenbaum N.: A Cox process involved in the Bose-Einstein condensation. Ann. Henri Poincaré 9, no. 6, 1123-1140 (2008). MR-2453257

[6] Eisenbaum N.: Permanental vectors and selfdecomposability. High dim probability VII, Progress in Probab., vol. 71, 359-362. Birkhauser (2016). MR-3565271

[7] Eisenbaum N. and Kaspi H.: On permanental processes. Stoch. Proc. and Appl. 119, no. 5, 1401-1415 (2009). MR-2513113

[8] Eisenbaum N., Kaspi H., Marcus M., Rosen J. and Shi Z.: A Ray-Knight Theorem for symmetric Markov processes. Annals of Probability 28, 1781-1796 (2000). MR-1813843

[9] Eisenbaum N. and Maunoury F.: Existence conditions of permanental and multivariate negative binomial distributions. Ann. Probab. no. 6B, 4786-4820 (2017). MR-3737924

[10] Fitzsimmons P. J. and Rosen J.: Markovian loop soups: permanental processes and isomorphism theorems. Electron. J. Probab. 19, no. 60, 1-30 (2014). MR-3238780

[11] Jain N. and Kallianpur G.: Oscillation function of a multiparameter Gaussian process. Nagoya Math. J. 47, 15-28 (1972). MR-0317399

[12] Kogan H. and Marcus M. B.: Permanental vectors. Stoch. Proc. Appl. 122, no. 4, 1226-1247 (2012). MR-2914751

[13] Marcus, M. B. and Rosen, J.: Necessary and sufficient conditions for the continuity of permanental processes associated with transient Lévy processes. Electron. Commun. Probab. 20, no. 57, 6 pp. (2015). MR-3384115

[14] Marcus M. and Rosen J.: Markov Processes, Gaussian Processes and Local Times. Cambridge University Press (2006). MR-2250510 
Infinitely divisible processes

[15] Pitman J. and Yor M.: A decomposition of Bessel bridges. Z. Wahrscheinlichkeitstheorie verw. Gebiete 59, 425-457 (1982). MR-0656509

[16] Rosinski J.: Representations and isomorphism identities for infinitely divisible processes. Ann. Probab. 46, no. 6, 3229-3274 (2018). MR-3857855

[17] Royden H. L. and Fitzpatrick P. M.: Real Analysis, fourth edition. The Macmillan Company (2010) (first edition MR-1013117)

Acknowledgments. We are grateful to Haya Kaspi and Jan Rosinski for our stimulating discussions, with special thanks to Haya for her useful comments on the first version of this work. 


\section{Electronic Journal of Probability Electronic Communications in Probability}

\section{Advantages of publishing in EJP-ECP}

- Very high standards

- Free for authors, free for readers

- Quick publication (no backlog)

- Secure publication $\left(\mathrm{LOCKSS}^{1}\right)$

- Easy interface (EJMS²)

\section{Economical model of EJP-ECP}

- Non profit, sponsored by $\mathrm{IMS}^{3}, \mathrm{BS}^{4}$, ProjectEuclid ${ }^{5}$

- Purely electronic

\section{Help keep the journal free and vigorous}

- Donate to the IMS open access fund ${ }^{6}$ (click here to donate!)

- Submit your best articles to EJP-ECP

- Choose EJP-ECP over for-profit journals

\footnotetext{
${ }^{1}$ LOCKSS: Lots of Copies Keep Stuff Safe http://www. lockss.org/

${ }^{2}$ EJMS: Electronic Journal Management System http://www.vtex.lt/en/ejms.html

${ }^{3}$ IMS: Institute of Mathematical Statistics http://www.imstat.org/

${ }^{4}$ BS: Bernoulli Society http://www. bernoulli-society.org/

${ }^{5}$ Project Euclid: https://projecteuclid.org/

${ }^{6}$ IMS Open Access Fund: http://www.imstat.org/publications/open.htm
} 